Draft VERSion July 10, 2018

Preprint typeset using $\mathrm{IAT}_{\mathrm{E}} \mathrm{X}$ style emulateapj v. 5/2/11

\title{
THE SCALING RELATIONS AND STAR FORMATION LAWS OF MINI-STARBURST COMPLEXES
}

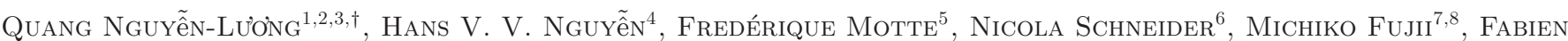 \\ Louvet $^{9}$, Tracey Hill ${ }^{10}$, Patricio Sanhueza ${ }^{1}$, James O. Chibueze ${ }^{11}$, and Pierre Didelon ${ }^{12}$ \\ Draft version July 10, 2018
}

\begin{abstract}
The scaling relations and the star formation laws for molecular cloud complexes in the Milky Way is investigated using data from the ${ }^{12} \mathrm{CO} 1-0$ CfA survey and from the literatures. We compare their masses $M_{\text {gas }}$, mass surface densities $\Sigma_{M_{\text {gas }}}$, radii $R$, velocity dispersions $\sigma$, star formation rates $S F R$, and SFR densities $\Sigma_{\text {SFR }}$ with those of structures ranging from cores, clumps, Giant Molecular Clouds (GMCs), to Molecular Cloud Complexes (MCCs), and to Galaxies, spanning 8 orders of magnitudes in size and 13 orders of magnitudes in mass. MCC are mostly large $(R>50$ pc $)$, massive $\left(\sim 10^{6} \mathrm{M}_{\odot}\right)$ gravitationally unbound cloud structures. This results in the following universal relations:
\end{abstract}

$$
\sigma \sim R^{0.5}, M_{\mathrm{gas}} \sim R^{2}, \Sigma_{\mathrm{SFR}} \sim \Sigma_{M_{\mathrm{gas}}}^{1.5}, S F R \sim M_{\mathrm{gas}}{ }^{0.9}, \text { and } S F R \sim \sigma^{2.7} .
$$

Variations in the slopes and the coefficients of these relations are found at individual scales signifying different physics acting at different scales. Additionally, there are breaks at the MCC scale in the $\sigma-R$ relation and between the starburst and the normal star-forming objects in the $S F R-M_{\text {gas }}$ and $\Sigma_{\mathrm{SFR}}-\Sigma_{\mathrm{M}_{\text {gas }}}$ relations. Therefore, we propose to use the Schmidt-Kennicutt diagram to distinguish the starburst from the normal star-forming structures by applying a $\Sigma_{M_{\text {gas }}}$ threshold of $\sim 100 \mathrm{M}_{\odot} \mathrm{pc}^{-2}$ and a $\Sigma_{\text {SFR }}$ threshold of $1 \mathrm{M}_{\odot} \mathrm{yr}^{-1} \mathrm{kpc}^{-2}$. Mini-starburst complexes are gravitationally unbound MCCs that have enhanced $\Sigma_{\mathrm{SFR}}\left(>1 \mathrm{M}_{\odot} \mathrm{yr}^{-1} \mathrm{kpc}^{-2}\right)$, probably caused by dynamic events such as radiation pressure, colliding flows, or spiral arm gravitational instability. Because of the dynamical evolution, gravitational boundedness does not play a significant role in characterizing the star formation activity of MCCs, especially the mini-starburst complexes, which leads to the conclusion that the formation of massive stars and clusters is dynamic. We emphasize the importance of understanding mini-starburst in investigating the physics of starburst galaxies.

Subject headings: stars: formation, ISM: clouds, ISM: structure, (ISM:) evolution, methods: observational, Galaxy: evolution

(Submitted on May 02, 2016; Accepted to publish on ApJ on Oct 09, 2016)

\section{INTRODUCTION}

${ }^{1}$ NAOJ Chile Observatory, National Astronomical Observatory of Japan, 2-21-1 Osawa, Mitaka, Tokyo 181-8588, Japan

2 Korea Astronomy and Space Science Institute, 776 Daedeok daero, Yuseoung, Daejeon 34055, Republic of Korea

${ }^{3}$ CITA, University of Toronto, 60 St. George Street, Toronto, ON M5S 3H8, Canada

${ }^{4}$ Max-Planck-Institut für Radioastronomie, Auf dem Hügel 69, D-53121 Bonn, Germany

${ }^{5}$ Institut de Planétologie et d'Astrophysique de Grenoble, LAOG (UMR 5571), Universit de Grenoble, BP 53, F-38041 Grenoble Cedex 09, France

${ }^{6}$ I. Physik. Institut, University of Cologne, 50937 Cologne, Germany

7 Theoretical Astronomy Division, National Astronomical Observatory of Japan, 2-21-1 Osawa, Mitaka, Tokyo 181-8588, Japan

8 Department of Astronomy, The University of Tokyo, Hongo 7-3-1, Bunkyo-ku, Tokyo, 113-0033, Japan

${ }^{9}$ Departamento de Astronomía, Universidad de Chile, Santiago, Chile

${ }^{10}$ Joint ALMA Observatory, 3107 Alonso de Cordova, Vitacura, Santiago, Chile

${ }^{11}$ Department of Physics and Astronomy, Faculty of Physical Sciences, University of Nigeria, Carver Building, 1 University Road, Nsukka, Nigeria

12 Laboratoire AIM Paris-Saclay, CEA/IRFU - CNRS/INSU - Université Paris Diderot, Service d'Astrophysique, Bât. 709, CEA-Saclay, F-91191, Gif-sur-Yvette Cedex, France

$\dagger$ EACOA Fellow at NAOJ, Japan \& KASI, Korea, quang.nguyen-luong@nao .ac.jp, quangnguyenluong@kasi.re.kr
Molecular gas is an indispensable element of the galactic ecological system and exists as coherent cloudy structures having different sizes: core $(<0.1 \mathrm{pc})$, clump $(0.1-$ $1 \mathrm{pc}$ ), molecular cloud (GMC, 1-10 pc), and molecular cloud complex (MCC, 10-100 pc) (Blitz \& Williams 1999). They are the stellar nursery, therefore, large surveys of molecular clouds in the Milky Way are necessary to understand their global star formation activities and connection to the global properties of the Galaxy.

The birth of the millimeter-wave radio astronomy, and subsequently, of the observations of Carbon Monoxide (CO) emission opened a new window into the molecular gas (Wilson et al. 1970). Since then, molecular gas was discovered progressively in star forming regions (Lada et al. 1974), in the Galaxy's diffuse interstellar medium (Cohen et al. 1980), and in other galaxies (Elmegreen et al. 1980), thus revealed the ubiquity of molecular gas.

A milestone of the wide-field molecular gas observation is the CO 1-0 almost-all-sky survey performed by the CfA $1.2 \mathrm{~m}$ telescopes (Dame et al. 1986, 2001). This survey formed a coherent and high spectral resolution basis for subsequent follow-up surveys. Since then, surveys of different excitation transitions or isotopologues were performed with larger telescopes to reach higher angular 
resolutions, for example: CO 1-0 from the Three-mm Ultimate Mopra Milky Way Survey (THRUMMS, Barnes et al. 2015), CO 3-2 from the CO 3-2 High-resolution Survey of the Galactic Plane (COHRS, Dempsey et al. 2013), or the ${ }^{13}$ CO 1-0 from the Galactic Ring Survey (GRS, Jackson et al. 2006). However, the CfA survey is still pertinent to create a large catalog of GMCs and MCCs, and to study the mutual scaling relations between different physical properties such as mass, size, velocity dispersion, and star formation rate (SFR).

The existence of the universal relations between different physical properties of star-forming structures is a suggestion that the basic physics governing different objects are similar and only scale with their sizes. Confirmation or refutation of this argument needs an investigation of these relations on datasets that cover the entire physical scale range. Early on, Larson (1981) pioneered in deriving the universal scaling relations between mass $M$, radius $R$, and velocity dispersion $\sigma$ of molecular clouds; later, they are named as the Larson's relations. First, the linewidth-size relation $\sigma \propto R^{\beta}$ with $\beta=0.38$ describes the structure of molecular clouds as fragmentation due to Kolmogorow-like turbulent cascade. Second, a linear correlation between the virial mass and total mass predicts the virial equilibrium state of GMCs. Third, the inverse relationship between the mean density and size implies a mass-size relation $M \propto R^{\alpha}$ with $\alpha=2$.

However, there are arguments against the universal scaling relations. Fore instance, Lombardi et al. (2010) and Kauffmann et al. (2010) derived a $M \propto R^{\alpha}$ relation with $\alpha \sim 1.2-1.6$ for substructures inside individual GMC. Heyer et al. (2009) suggested that the coefficient of the velocity dispersion-radius relation for Galaxy's GMCs scales with the surface density. Hughes et al. (2013) found no trivial scaling relations between the three quantities: mass, size and velocity dispersion for GMCs and MCCs in M51, M33, and the Large Magellanic Cloud. Utomo et al. (2015) and Rebolledo et al. (2015) found similar results for GMCs with average radii of 20 pc in NGC 4526 and MCCs with averaged radii of $\sim 140-180 \mathrm{pc}$ in nearby galaxies, respectively. Furthermore, Schneider \& Brooks (2004) showed that the coefficients of these relations strongly depend on the cloud structure identification algorithm that was used.

Likewise, Schmidt (1959) and Kennicutt (1998) found a correlation between gas surface density, $\sigma$, and Star Formation Rate density, $\Sigma_{\mathrm{SFR}}$, in the form of $\Sigma_{\mathrm{SFR}}=$ $A_{\mathrm{KS}} \times \sigma^{N}$. Galaxy types, spatial resolutions, SFR tracers, and gas tracers are among the multiple factors that can change the power law index $N$ and the normalization factor $A_{\mathrm{KS}}$ (Deharveng et al. 1994; Gao \& Solomon 2004; Bigiel et al. 2008; Daddi et al. 2010).

The recent combinations of observations of Galactic clouds of $\sim 1-10 \mathrm{pc}$ and galaxy populations of $\sim 1-10 \mathrm{kpc}$ revealed a large spread in the $\Sigma_{S F R}-\sigma$ diagram (Heiderman et al. 2010; Evans et al. 2014; Willis et al. $2015)$. For example, the $\Sigma_{S F R}-\sigma$ relation of low-mass Galactic clouds derived by Heiderman et al. (2010) has a steeper slope than the extragalactic one derived by Kennicutt (1998). Power law indexes within the massive star forming complexes were found to scatter from 1.7 to 2.8 (Willis et al. 2015). Various modifications of the Schmidt-Kennicutt relation were proposed: nor- malizing $\sigma$ by dynamical (Daddi et al. 2010) or freefall timescales (Krumholz et al. 2012a), considering the SFRdense gas relation as $\Sigma_{\mathrm{SFR}} \propto \Sigma_{\text {dense gas }}$ (Lada et al. 2012; Evans et al. 2014), or replacing the surface density by volume density quantities to produce a linear relation between star formation rate volume-density, $\rho_{S F R}$, and mass volume-density, $\rho_{\text {gas }}$ (Evans et al. 2014).

Studies trying to link these scaling relations across different spatial scales, from local Galactic clouds to global galaxy, seem to miss the MCC population, also called Giant Molecular Associations, having sizes and masses of $>50 \mathrm{pc}$ and $\sim 10^{6}-10^{7} \mathrm{M}_{\odot}$, respectively (Nguyen Luong et al. 2011b). MCCs are important as they are the largest cloud agglomeration in a galaxy and massive star formation is linked with a special category of MCCs, the mini-starburst complexes (e.g., Motte et al. 2003; Louvet et al. 2014). They are the birthplaces of massive OB stars and Young Massive Clusters (YMCs), thus are important in maintaining the chemical, energy, and mass balance of hosting galaxies (Bressert et al. 2012). This is especially true for starburst galaxies such as the Antennae merger system, which undergoes starburst events and contains many YMCs and mini-starburst MCCs (Herrera et al. 2012; Whitmore et al. 2014).

Motivated by the needs of quantifying the scaling relations between different physical quantities of MCCs in the Milky Way, we use the CfA CO survey to catalog and characterize the physical properties of our MCCs and use radio continuum data to measure their SFR. We discuss the data and the method of identifying sources in Sections 2 and 3. In Section 4, we derive the physical properties and SFR (density) of MCCs. Section 5 and Section 6 will examine the scaling relations between different cloud properties and the star formation laws for the all cloud structures ranging from GMCs to Galaxies. We elaborate more the division of (mini)starburst and normal-star forming objects by using the SchmidtKennicutt diagram and the possible sequence of forming mini-starburst in Section 7.

\section{DATA \\ 2.1. ${ }^{12}$ CO Data from the CfA Survey}

The ${ }^{12} \mathrm{CO} 1-0$ data from the CO all-sky survey is used to catalog and characterize MCCs in the Galaxy. This survey was made with the CfA $1.2 \mathrm{~m}$ telescopes located in both northern and southern hemispheres. The observations are sub-Nyquist sampled with an effective angular resolution of $8^{\prime} .8$. The spectral cube contains data obtained from various independent observations starting in 1986 (Dame et al. 1986; Bronfman et al. 1989) and ending in 2001 (Dame et al. 2001), stored in the CO survey archive $^{14}$. The combined data cube is provided in main beam antenna temperature and has a sensitivity of $\sim 1.5$ $\mathrm{K}$ per $0.65 \mathrm{~km} \mathrm{~s}^{-1}$, although individual surveys have sensitivity $\sim 0.12-1 \mathrm{~K}$ per $0.65-\mathrm{km} \mathrm{s}^{-1}$ channel. We use the "whole galaxy cube" covering the entire $360^{\circ}$ longitude range and $-40^{\circ}$ to $40^{\circ}$ latitude range. The integrated map of the Galactic Plane, where MCCs reside, is shown in Figure 1.

\subsection{Radio continuum}

14 http://www.cfa.harvard.edu/rtdc/CO/ 
To derive the SFRs of MCCs, we use the $21 \mathrm{~cm}$ radio continuum data from the VLA Galactic Plane Survey (VGPS, Stil et al. 2006), the Canadian Galactic Plane Survey (CGPS, Taylor et al. 2003), and the Southern Galactic Plane Survey (SGPS, Haverkorn et al. 2006). VGPS has a FWHM beam of $1^{\prime}$ and covers the Galactic longitude $18-67^{\circ}$, CGPS has a FWHM beam of $1^{\prime}$ and covers the Galactic longitude $63-175^{\circ}$, and SGPS has a FWHM of $2.2^{\prime}$ covering the Galactic longitude $253-358^{\circ}$.

\subsection{Complementary data}

We complement our data with literature data to increase the dynamical ranges of all parameters. For the Larson relations (Section 5), we add cloud properties data from the following sources:

- clumps/GMCs: Maruta et al. (2010), Onishi et al. (2002), Shimajiri et al. (2015), Heyer et al. (2009), Roman-Duval et al. (2010), Evans et al. (2014).

- MCCs: García et al. (2014), Murray (2011), Donovan Meyer et al. (2013), Rosolowsky (2007), Miura et al. (2012, 2014), Wei et al. (2012).

- Galaxies: Leroy et al. (2013), Tacconi et al. (2013), Genzel et al. (2010).

For the Schmidt-Kennicutt scaling relation (Section 6), we add SFR data from the following sources:

- GMCs: Heiderman et al. (2010), Lada et al. (2010), Evans et al. (2014).

- MCCs: García et al. (2014), Bolatto et al. (2008), Murray (2011), Donovan Meyer et al. (2013), Rosolowsky (2007), Miura et al. (2012, 2014), Wei et al. (2012).

- Galaxies: Leroy et al. (2013), Tacconi et al. (2013), Genzel et al. (2010).

For the galaxies sample, we calculate the velocity dispersion assuming that the entire galaxy is a dynamical system, thus we do not distinguish between elliptical and disk galaxies or starburst and normal galaxies. We convert the orbital time from Leroy et al. (2013) sample or the dynamical time from Genzel et al. (2010) and Tacconi et al. (2013) samples to a rotation velocity as $V_{\text {rot }}=\frac{2 \pi R}{t_{\mathrm{dyn}, \text { orb }}}$. Then, the velocity dispersion is written as $\sigma=\frac{2 \pi^{2} G R \sigma}{1.5 V_{\text {rot }}}$. The radi used here are either the optical B-band 25th magnitude isophote (Leroy et al. 2013) or the half-light radii (Genzel et al. 2010; Tacconi et al. 2013). The local galaxies in Leroy et al. (2013) have velocity dispersions smaller than their rotation velocity, but the high-z galaxies in Genzel et al. (2010); Tacconi et al. (2013) have velocity dispersion larger than rotation velocity. This is probably, because the high-z galaxies are highly turbulent than the local galaxies.

Beside that, we also add data from individual ministarburst regions in a galaxy at $z=1.987$ (Zanella et al. 2015), in SDP81 galaxy at $z=3.042$ (Hatsukade et al. 2015), and in Arp 220 (Scoville 2013).

Systematic errors caused by different measurements are unavoidable but we assume that these errors have little affect on our analysis. For example, errors caused by mass determinations from different tracers such as ${ }^{12} \mathrm{CO},{ }^{13} \mathrm{CO}$, or dust emission; or errors caused by the variation of the abundances for the $\mathrm{CO} / \mathrm{H}_{2}$ conversion as a function of position in the galaxy; or errors caused by the exclusion of the CO-dark gas, are not taken into account. So, the mass estimates are the lower limits of the true values. We are aware of these error sources, but due to our large statistical sample, and assuming an uncertainty of typically $50 \%$, we are still able to derive robust scaling relations. Actually, these errors can be safely ignored because they typically vary around about $30 \%-100 \%$ of the measured values and all relations that we will discuss are presented in log-log space.

\section{SOURCE IDENTIFICATION AND DISTANCES}

Initially, we use the Duchamp ${ }^{15}$ algorithm to decompose the $3 \mathrm{D}$ spectral cube. This program is designed to extract sources in large $\mathrm{H}$ I surveys and is the main extraction tool of the Australian Square Kilometre Array Pathfinder (Whiting \& Humphreys 2012). Although it is optimized for $\mathrm{H}$ I source extraction, its utility towards molecular clouds and maser extraction has been proven (Carlhoff et al. 2013; Walsh et al. 2016). For our extraction, Duchamp detected successfully GMCs outside and inside the Galactic Plane. However, in the Galactic Plane, Duchamp tends to either break down the emission into too many individual clouds if we set a weak merging condition or merge to too large structures. This is likely the same problem occured for other detection alogrithms (Gaussclumps, Stutzki \& Guesten 1989; Clumpfind, Williams et al. 1994; Dendogram, Rosolowsky et al. 2008 ). Finally, we decided for a conservative approach to select MCCs by eye inspection.

We identify strong $\mathrm{CO}$ peaks in the integrated intensity and position-velocity maps of Dame et al. (2001) and then average $\mathrm{CO}$ spectra over $0.5^{\circ} \times 0.5^{\circ}$ areas surrounding the $\mathrm{CO}$ peaks that correspond to $\sim 13-52 \mathrm{pc}$ boxes at $1.5-6 \mathrm{kpc}$ distances. The global velocity extent measured from these first-guess integrated spectra are used to integrate $\mathrm{CO}$ lines and create first-guess integrated maps of the MCC. For each MCC, we then iteratively refine its associated area, $A$, and velocity extent until they are properly distinguished both in velocity and space from a background of three times the local rms. By setting the local rms as threshold, we can trace all material down to the observational sensitivity level, but not having homogeneous column density threshold across all MCCs. We fit Gaussian profiles to the average spectrum of each MCC to derive its velocity dispersion, $\sigma_{\mathrm{CO}}$, and its velocity-integrated intensity, $W_{\mathrm{CO}}$. To summarize, the main criteria to include a cloud in MCC are that their velocity range is not more than $15 \mathrm{~km} \mathrm{~s}^{-1}$ far from the bulk velocity of the cloud and it is connected to the main and other clouds by diffuse gas features. This method was proven to be more suited to identify MCCs than automatic detection, as in the cases of the W43 (Nguyen Luong et al. 2011a) and the RCW 106 (Nguyen et al. 2015).

After identifying a sample of 44 MCCs (Table 1), we estimate their distances to the Sun following this sequential scheme: (1) assigning a parallax distance if it is available from the Bar and Spiral Structure Legacy (BeSSeL,

\footnotetext{
15 http://www.atnf.csiro.au/people/Matthew.Whiting/Duchamp
} 


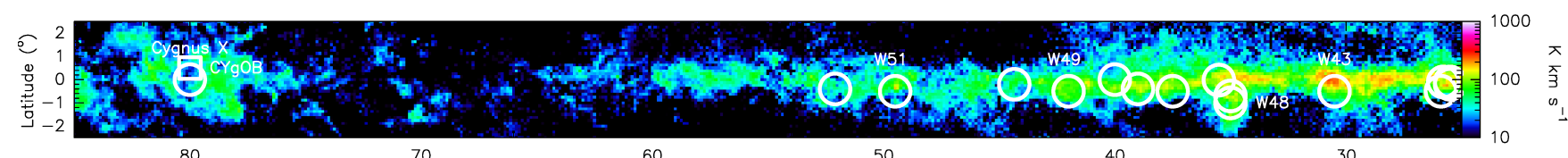

80

60

50

40

30

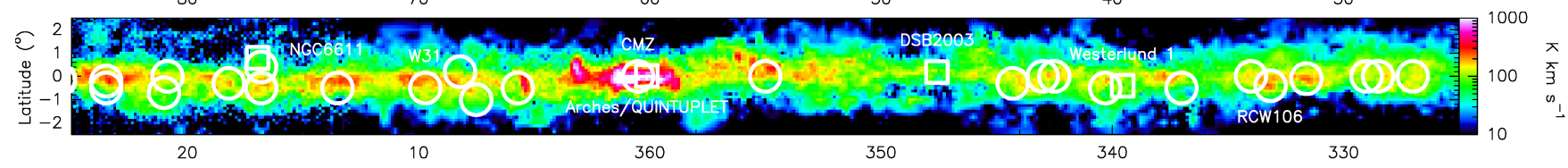

20

360

350

340

330

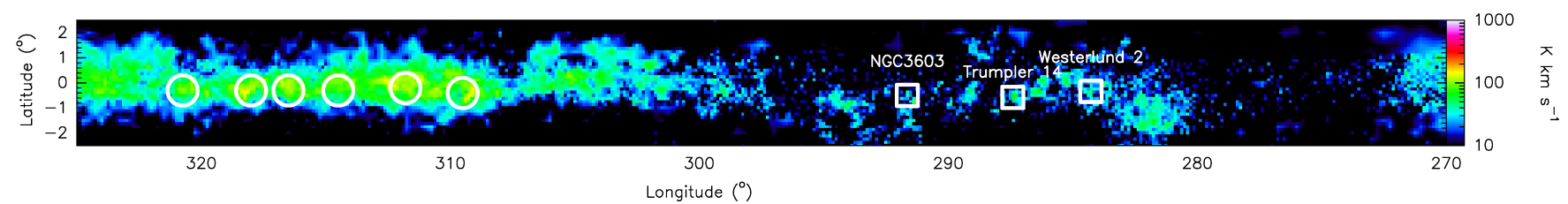

Figure 1. The integrated ${ }^{12} \mathrm{CO} 1-0$ map of the CO CfA all sky survey, over which mini-starburst MCCs are indicated by circles. Names of well-known MCCs are labelled. Young Massive Clusters and OB Associations from Portegies Zwart et al. (2010) are marked as squares, respectively.

Reid et al. 2014) ${ }^{16}$ and the VLBI Exploration of Radio Astrometry (VERA, Honma et al. 2007) ${ }^{17}$ projects or other individual parallax measurements from the literature; (2) assigning a photometric distance if it is available from the literature; (3) assigning an averaged ambiguityresolved kinematic distance if it is available from the literature; (4) calculating our own kinematic distance as $d_{\text {kin }}=R_{0} \cos (l) \pm \sqrt{r^{2}-R_{0}^{2} \sin ^{2}(l)}$ where $R_{0}$ is the Galactocentric radius of the Sun, $V_{0}$ is the orbital velocity of the Sun around the Galactic center, $V(r)$ is the rotation curve and $V_{\mathrm{r}}$ is the radial velocity of the cloud. The result is 11 out of 44 have parallax distances, 1 has photometric distance, 8 have kinematic distances with ambiguity resolved by $\mathrm{HI}$ absorption, and 24 have near kinematic distances.

\section{PHYSICAL PROPERTIES}

\subsection{Cloud properties}

We use the CO 1-0 emission as a proxy to estimate the total gas mass content of molecular cloud structure. For this purpose, using the CO 1-0 alone is subjected to two major problems. First, $\mathrm{CO}$ may not trace all molecular hydrogen in molecular clouds and it miss the 'CO-dark' gas (Langer et al. 2014). Second, the CO 10 emission becomes optically thick quickly in the dense parts of the molecular clouds. Moreover, Barnes et al. (2015) proposed that the linear conversion from CO integrated intensity to $\mathrm{H}_{2}$ gas column density might underestimate the true column density. However, for the first-order global mass estimate, this is acceptable. The $\mathrm{X}$ factor $X=2 \times 10^{20} \mathrm{~cm}^{-2} \mathrm{~K}^{-1}\left(\mathrm{~km} \mathrm{~s}^{-1}\right)^{-1}$ is used to convert the $\mathrm{CO}$ integrated intensity to $\mathrm{H}_{2}$ gas column density as recommended by Bolatto et al. (2013), which was established after an exhausted investigation of all possible measurements. The mean hydrogen molecular mass $m_{\mathrm{H}_{2}}=\mu m_{\mathrm{H}}=2.8 m_{\mathrm{H}}$ which also accounts for helium contained in the gas is used to convert from number to mass column density. We note that additionally intrinsic uncertainties on the $X$ factor can also contribute to the error of the estimated column density and mass (see, e.g., Shetty et al. 2011).

\footnotetext{
16 http://bessel.vlbi-astrometry.org

17 http://veraserver.mtk.nao.ac.jp
}

Eventually, we derived the following physical parameters:

- Velocity-integrated intensity $W_{\mathrm{CO}}\left(\mathrm{K} \mathrm{km} \mathrm{s}^{-1}\right)$ by integrating the channel maps within the velocity range derived from the Gaussian fit of the integrated spectrum,

- Equivalent radius $R=\sqrt{A / \pi}$ (pc) from the surface area $A\left(\mathrm{pc}^{2}\right)$ measured in the integrated map,

- Velocity dispersion $\sigma=\frac{\Delta v, \text { FWHM }}{\sqrt{8 \ln 2}}\left(\mathrm{~km} \mathrm{~s}^{-1}\right)$ from the FWHM linewidth $\Delta v_{\text {,FWHM }}$ resulted from Gaussian fitting of the integrated spectra,

- CO luminosity $L_{\mathrm{CO}}=A W_{\mathrm{CO}}\left(\mathrm{K} \mathrm{km} \mathrm{s}^{-1} \mathrm{pc}^{2}\right)$,

- Total gas mass $M=L_{\mathrm{CO}} X_{\mathrm{CO}} m_{\mathrm{H} 2}\left(\mathrm{M}_{\odot}\right)$,

- Gas surface density $\Sigma_{\text {gas }}=\frac{M}{A}\left(\mathrm{M}_{\odot} \mathrm{pc}^{-2}\right)$,

- Virial parameter $\alpha_{\mathrm{vir}}=5 \sigma_{1 \mathrm{D}}^{2} R / G M$ with the gravitational constant $\mathrm{G}$.

Our focus is on the 44 MCCs that are more massive than $10^{6} \mathrm{M}_{\odot}$ (Table 1). All of them lie in the Galactic Plane within the longitude ranging from $0^{\circ}$ to $90^{\circ}$ or $310^{\circ}$ to $355^{\circ}$ and the latitude ranging from $1^{\circ}$ to $+1^{\circ}$, thus, mainly in the first and fourth quadrants (see Figure 1). Our MCCs coincide spatially with all massive GMCs having mass larger than $10^{6} \mathrm{M}_{\odot}$ in the other CO surveys (Heyer et al. 2009; Roman-Duval et al. 2010; García et al. 2014). All MCCs hosting massive star clusters characterized by Murray (2011) are associated with MCCs in our catalog. We also cover wellknown MCCs such as W43 (Nguyen Luong et al. 2011a), Cygnus X (Schneider et al. 2006), W49 (Galván-Madrid et al. 2013), and W51 (Ginsburg et al. 2015). Therefore, we can conclude that our MCCs catalog is a robust catalog of nearby MCCs. Table 1 lists, for each MCC the location (l, b, $\left.V_{\mathrm{VLSR}}\right)$, extent $(A, R, \sigma)$, CO luminosity $\left(L_{\mathrm{CO}}\right)$ along with its associated mass $\left(M_{\text {gas }}\right)$, gas surface density $\left(\Sigma_{\text {gas }}\right)$ and viral parameter $\left(\alpha_{\text {vir }}\right)$. Their typical sizes and masses range from $40 \mathrm{pc}$ to $100 \mathrm{pc}$ and from $1 \times 10^{6} \mathrm{M}_{\odot}$ to $5 \times 10^{7} \mathrm{M}_{\odot}$. One exception is the Central 


\begin{tabular}{|c|c|c|c|c|c|c|c|c|c|c|c|c|c|c|}
\hline $\begin{array}{l}\text { Complex } \\
\text { name }\end{array}$ & $\begin{array}{r}1 \\
\left({ }^{\circ}\right) \\
\end{array}$ & $\begin{array}{r}\mathrm{b} \\
\left({ }^{\circ}\right) \\
\end{array}$ & $\begin{array}{c}V_{\mathrm{LSR}} \\
\left(\mathrm{km} \mathrm{s}^{-1}\right) \\
\end{array}$ & $\left(\mathrm{km} \mathrm{s}^{-1}\right)$ & $\begin{array}{c}d \\
(\mathrm{kpc}) \\
\end{array}$ & $\begin{array}{r}A \\
\times 10^{-2} \\
\left(\mathrm{kpc}^{2}\right) \\
\end{array}$ & $\begin{array}{r}R \\
(\mathrm{pc}) \\
\end{array}$ & $\begin{array}{l}L_{\mathrm{CO}} \\
\times 10^{3} \\
\left(\mathrm{~L}_{\odot}\right) \\
\end{array}$ & $\begin{array}{r}M_{\text {gas }} \\
\times 10^{6} \\
\left(\mathrm{M}_{\odot}\right) \\
\end{array}$ & $\begin{array}{c}\Sigma_{\text {gas }} \\
\left(\mathrm{M}_{\odot} \mathrm{pc}^{-2}\right) \\
\end{array}$ & $\begin{array}{c}S_{21 \mathrm{~cm}}^{\text {int }} \\
(\mathrm{Jy}) \\
\end{array}$ & $\begin{array}{c}\mathrm{SFR} \\
\left(\mathrm{M}_{\odot} \mathrm{yr}^{-1}\right) \\
\end{array}$ & $\begin{array}{c}\Sigma_{\mathrm{SFR}} \\
\left(\mathrm{M}_{\odot} \mathrm{yr}^{-1} \mathrm{kpc}^{-2}\right) \\
\end{array}$ & $\alpha_{\mathrm{vir}}$ \\
\hline G111 & 111.0 & -1.0 & -49 & 7.9 & 3.34 & 5.7 & 134 & 7.2 & 2.2 & 38.7 & 1860 & 0.014 & 0.25 & 4.4 \\
\hline G80-CygnusX & 80.0 & 0.0 & 2 & 6.6 & 1.5 & 3.7 & 108 & 40.7 & 2.2 & 59.9 & 45759 & 0.062 & 1.68 & 2.5 \\
\hline G52..... & 52.1 & -0.4 & 55 & 6.9 & 6.9 & 2.0 & 79 & 1.8 & 1.9 & 95.6 & 2818 & 0.0725 & 3.6 & 2.3 \\
\hline G49-W51.... & 49.5 & -0.5 & 57 & 9.8 & 5.41 & 1.8 & 76 & 3.6 & 2.5 & 139.9 & 10237 & 0.184 & 10.22 & 3.4 \\
\hline G44.4..... & 44.4 & -0.2 & 61 & 7.2 & 9.3 & 1.8 & 75 & 2.7 & 1.5 & 82.7 & 5272 & 0.070 & 3.89 & 3.1 \\
\hline G42-W49.... & 42.0 & -0.5 & 63 & 9.5 & 11.1 & 2.8 & 93 & 1.5 & 4.9 & 179.2 & 6069 & 0.51 & 17.86 & 2.0 \\
\hline G40....... & 40.0 & 0.0 & 32 & 6.1 & 2.2 & 3.4 & 104 & 13.0 & 1.8 & 52.1 & 7783 & 0.026 & 0.77 & 2.5 \\
\hline G39....... & 39.0 & -0.4 & 65 & 27.3 & 12.1 & 13.1 & 204 & 33.1 & 15.7 & 120.2 & 6447 & 0.079 & 0.60 & 11.3 \\
\hline G35-W48.... & 35.0 & -1.0 & 46 & 14.0 & 3.27 & 3.3 & 101 & 15.3 & 4.5 & 138.0 & 8379 & 0.075 & 2.27 & 5.2 \\
\hline G30-W43.... & 30.5 & -0.5 & 93 & 16.3 & 5.5 & 2.8 & 94 & 11.1 & 9.3 & 329.5 & 15282 & 0.320 & 11.40 & 3.1 \\
\hline G25.9.... & 25.9 & -0.5 & 100 & 10.0 & 5.4 & 2.5 & 88 & 4.5 & 3.7 & 148.5 & 12688 & 0.255 & 10.24 & 2.8 \\
\hline $\mathrm{G} 25.5+52 \mathrm{~km} \mathrm{~s}^{-1}$ & 25.5 & -0.2 & 52 & 11.1 & 3.4 & 1.1 & 58 & 5.2 & 1.7 & 154.0 & 15012 & 0.120 & 10.91 & 5.0 \\
\hline $\mathrm{G} 25.5+102 \mathrm{~km} \mathrm{~s}^{-1}$ & 25.5 & -0.1 & 102 & 11.8 & 5.5 & 2.7 & 93 & 5.6 & 4.6 & 169.8 & 15164 & 0.317 & 11.75 & 3.2 \\
\hline $\mathrm{G} 23.7+60 \mathrm{~km} \mathrm{~s}^{-1}$ & 23.7 & -0.5 & 60 & 9.9 & 6.21 & 2.0 & 80 & 4.4 & 4.7 & 230.9 & 15342 & 0.407 & 20.40 & 2.0 \\
\hline $\mathrm{G} 24+100 \mathrm{~km} \mathrm{~s}^{-1}$ & 23.5 & -0.2 & 93 & 21.1 & 5.9 & 3.2 & 101 & 13.9 & 13.2 & 411.9 & 17171 & 0.413 & 12.91 & 4.0 \\
\hline $\mathrm{G} 21 \ldots \ldots \ldots$ & 21.0 & -0.7 & 51 & 13.2 & 3.6 & 1.2 & 61 & 7.3 & 2.6 & 216.8 & 11690 & 0.1048 & 6.10 & 4.8 \\
\hline G20.9.... & 20.9 & 0.0 & 32 & 14.7 & 2.6 & 0.6 & 44 & 5.1 & 1.0 & 151.3 & 12909 & 0.060 & 10.00 & 11.8 \\
\hline $\mathrm{G} 18.2 \ldots \ldots$ & 18.2 & -0.3 & 47 & 10.3 & 3.6 & 1.2 & 61 & 7.3 & 2.6 & 217.7 & 5044 & 0.045 & 3.75 & 3.0 \\
\hline G16.8-M16/M17.... & 16.8 & 0.4 & 23 & 5.9 & 1.98 & 0.5 & 39 & 4.7 & 0.7 & 140.7 & 8560 & 0.031 & 6.20 & 2.3 \\
\hline G13.5-W33.. & 13.5 & -0.5 & 24 & 36.1 & 2.92 & 1.2 & 61 & 29.8 & 4.7 & 393.2 & 439 & 0.00175 & 0.149 & 19.8 \\
\hline G10-W31.... & 9.7 & -0.5 & 22 & 15.7 & 4.95 & 2.3 & 85 & 14.0 & 9.5 & 416.2 & - & - & 1.0 & 2.6 \\
\hline G8.2...... & 8.2 & 0.2 & 17 & 8.4 & 2.9 & 0.8 & 49 & 6.3 & 1.5 & 188.4 & - & - & 1.0 & 2.8 \\
\hline G7.5..... & 7.5 & -1.0 & 17 & 8.7 & 3.0 & 0.8 & 51 & 7.9 & 2.0 & 234.4 & - & - & 1.0 & 2.3 \\
\hline G3.5...... & 5.7 & -0.5 & 13 & 8.6 & 3.1 & 2.8 & 93 & 19.0 & 5.0 & 179.7 & - & - & 0.9 & 1.6 \\
\hline G0-CMZ..... & 0.5 & 0.0 & -6 & 23.1 & 7.9 & 51.6 & 405 & 74.8 & 128.5 & 249.1 & - & - & 0.9 & 2.0 \\
\hline G355...... & 355.0 & 0.0 & 95 & 20.0 & 6.1 & 0.8 & 50 & 2.7 & 2.8 & 344.2 & 824 & 0.0212 & 2.50 & 8.4 \\
\hline G344....... & 344.3 & -0.3 & -71 & 8.1 & 4.8 & 2.6 & 91 & 3.3 & 2.1 & 80.4 & 703 & 0.0112 & 0.423 & 3.3 \\
\hline G343....... & 343.0 & 0.0 & -28 & 8.0 & 2.7 & 2.6 & 91 & 17.5 & 3.4 & 129.7 & 426 & 0.00214 & 0.077 & 2.0 \\
\hline G342-127 km s${ }^{-1}$ & 342.5 & 0.0 & -127 & 8.1 & 8.10 & 3.5 & 105 & 2.2 & 2.5 & 71.3 & 245 & 0.007 & 0.17 & 3.2 \\
\hline G342-79 $\mathrm{km} \mathrm{s}^{-1}$ & 342.5 & 0.0 & -79 & 7.8 & 4.74 & 2.3 & 85 & 3.4 & 2.4 & 102.8 & 245 & 0.004 & 0.173 & 2.5 \\
\hline G340...... & 340.3 & -0.5 & -37 & 14.1 & 3.41 & 0.8 & 51 & 10.4 & 2.6 & 309.4 & 684 & 0.00426 & 0.50 & 4.6 \\
\hline G337...... & 337.0 & -0.5 & -118 & 8.9 & 7.85 & 3.8 & 109 & 2.3 & 2.9 & 76.5 & 2385 & 0.074 & 1.95 & 3.5 \\
\hline G334...... & 334.0 & 0.0 & -87 & 9.4 & 4.9 & 2.2 & 84 & 4.1 & 2.7 & 122.4 & 2312 & 0.0384 & 1.77 & 3.2 \\
\hline G330-RCW106 & 333.1 & -0.4 & -46 & 13.9 & 3.5 & 0.9 & 53 & 11.7 & 3.2 & 348.4 & 3111 & 0.0278 & 3.22 & 3.8 \\
\hline G331-90 $\mathrm{km} \mathrm{s}^{-1}$ & 331.6 & -0.1 & -93 & 10.4 & 7.44 & 2.5 & 88 & 5.5 & 4.0 & 162.0 & 1863 & 0.0348 & 1.40 & 2.8 \\
\hline G329-75 $\mathrm{km} \mathrm{s}^{-1} .$. & 328.5 & 0.0 & -75 & 30.2 & 4.4 & 4.1 & 114 & 28.0 & 15.1 & 369.5 & 714 & 0.0095 & 0.244 & 8.0 \\
\hline G329-25 $\mathrm{km} \mathrm{s}^{-1} .$. & 329.0 & 0.0 & -45 & 7.7 & 3.0 & 3.3 & 103 & 14.3 & 3.5 & 104.6 & 917 & 0.00571 & 0.18 & 2.0 \\
\hline G327...... & 327.0 & 0.0 & -45 & 7.8 & 2.9 & 1.8 & 75 & 11.3 & 2.74 & 149.4 & 2563 & 0.0149 & 0.83 & 2.0 \\
\hline G320...... & 320.8 & -0.3 & -62 & 12.3 & 4.0 & 1.5 & 69 & 4.2 & 1.9 & 124.1 & 811 & 0.00897 & 0.67 & 6.5 \\
\hline G318....... & 318.0 & -0.3 & -44 & 8.1 & 3.0 & 0.8 & 51 & 5.5 & 1.4 & 163.6 & 833 & 0.00518 & 0.625 & 2.9 \\
\hline G316.5.... & 316.5 & -0.3 & -48 & 9.1 & 3.3 & 1.0 & 57 & 6.1 & 1.9 & 180.0 & 1224 & 0.0092 & 0.1 & 3.0 \\
\hline G314...... & 314.5 & -0.3 & -49 & 8.9 & 3.6 & 1.2 & 61 & 7.4 & 2.6 & 219.3 & 280 & 0.0025 & 0.25 & 2.1 \\
\hline G311...... & 311.8 & -0.2 & -49 & 8.9 & 4.0 & 2.4 & 86 & 11.7 & 5.1 & 214.4 & 1099 & 0.0121 & 0.5 & 1.6 \\
\hline G309...... & 309.5 & -0.4 & -44 & 11.2 & 3.8 & 1.8 & 75 & 9.0 & 3.7 & 205.9 & 528 & 0.0053 & 0.28 & 3.0 \\
\hline
\end{tabular}

Table 1

Cloud and SFR characteristics of the massive molecular cloud complexes (MCCs) in the Milky Way. The distances quoted in red rectangles are parallax distances, in black rectangles are photometric distances, in green rectangles are ambiguity resolved kinematic distances, and the rests are near-kinematic distances. References of parallax distances to several well-known MCCs: G111 (Choi et al. 2014), Cygnus X (Rygl et al. 2012), W51 (Sato et al. 2010), W49 (Zhang et al. 2013),

G35-W48 (Zhang et al. 2009), W43 (Zhang et al. 2014), G23.7+60 (Sanna et al. 2014), M16/M17 (Xu et al. 2011 ), W33

(Immer et al. 2013), W31 (Sanna et al. 2014), CMZ (Reid et al. 2009), CMZ (Reid et al. 2009), NGC6334/NGC6357

(Chibueze et al. 2014). For ambiguity resolved kinematic distances, we obtain them from Jones \& Dickey (2012) and Anderson \& Bania (2009). The SFRS of G10, G8.2, G7.5, G3.5, and CMZ MCCs are not calculated because the lack of radio continuum data toward the central molecular zone. 
Molecular Zone which has a mass of $1.3 \times 10^{8} \mathrm{M}_{\odot}$ over a area with equivalent radius of $406 \mathrm{pc}$.

To assess the detection completeness, we compare the number of MCCs in our catalog (44) with the total expected number of MCCs in the Milky Way from powerlaw mass distribution, $\frac{d N}{d m} \propto m_{\text {GMC }}^{\gamma}$ with $\gamma=-1.5$ (e.g. Simon et al. 2001). Assuming that the Galactic molecular mass ranges from the minimum mass $M_{\mathrm{L}}=10^{2} \mathrm{M}_{\odot}$ to the maximum mass $M_{\mathrm{U}}=10^{7} \mathrm{M}_{\odot}$, we calculate the number of GMCs above a certain mass $m$ by integrating the mass distribution function over the mass range $\left[M_{\mathrm{L}}-m\right]$. This yields:

$$
N(>m)=\frac{2-\gamma}{\gamma-1} \times\left(\frac{\left(\frac{M_{\mathrm{U}}}{m}\right)^{\gamma-1}-1}{1-\left(\frac{M_{\mathrm{L}}}{M_{\mathrm{U}}}\right)^{2-\gamma}}\right) \times \frac{M_{\mathrm{Tot}}}{M_{\mathrm{U}}},
$$

where $M_{\text {Tot }}=10^{9} \mathrm{M}_{\odot}$ is the total gas mass in the Milky Way (Dame et al. 2001). A total number of $~ 300 \mathrm{MCCs}$ with masses larger than $10^{6} \mathrm{M}_{\odot}$ is expected in the entire Galaxy, about six times the number of detected MCCs. Our detection scheme mostly focuses on the near side of the Milky Way, covering only $\sim 1 / 5$ of the Galactic plane, the part in the first and fourth quadrants (see Figure 2) therefore the total number of $44 \mathrm{MCCs}$ is complete within this Galactic region.

MCCs scatter along the Galactic plane and follow closely the spiral arms structure (see Figures 1 and 2). Most (70\%) of the MCCs lie along the Scutum-Centaurus or Sagittarius arms, the two most prominent spiral arms in the Milky Way (Dame et al. 2001), and a few other lie along the Norma arm, Perseus arms, and interarm regions. We note that $\mathrm{YMCs}$, the potential descendant of mini-starburst clouds, also lie along the spiral arms (Portegies Zwart et al. 2010) (see also Figure 2). This is in line with extragalactic observations and numerical simulations, which find that the most massive, most turbulent, and most actively star-forming regions are located in the spiral arms rather than in the inter-arm zones (Koda et al. 2012; Dobbs et al. 2006; Fujimoto et al. 2014). MCCs are concentrated to the midplane, they distribute within the latitude range $\pm 1^{\circ}$ or approximately $\sim 200 \mathrm{pc}$ from the Galactic plane equator (see Figure 1).

\subsection{SFRs from radio continuum emission}

To calculate the immediate past (last $5 \times 10^{6} \mathrm{yr}$, or the timescale of one OB star generation) SFR of MCCs, we follow the same approach as in Nguyen et al. (2015). Following Mezger \& Henderson (1967), we use the $21 \mathrm{~cm}$ continuum emission to calculate the total Ly $\alpha$ continuum photons emitted by young massive stars that drive HiI regions as

$\frac{N_{\mathrm{Ly} \alpha}}{8.9 \times 10^{46} \mathrm{~s}^{-1}}=\frac{S_{\nu}}{\mathrm{Jy}}\left(\frac{\nu}{\mathrm{GHz}}\right)^{0.1}\left(\frac{T_{e}}{10^{4} \mathrm{~K}}\right)^{-0.45}\left(\frac{d}{\mathrm{kpc}}\right)^{2}$.

where $T_{e}=8000 \mathrm{~K}$ (Wilson et al. 2012) is the electron temperature, $\nu=1.42 \mathrm{GHz}$ is the observing frequency, and $d$ is the distance to the region.

Assuming that an O7 star with mass larger than $25 \mathrm{M}_{\odot}$ emits on average $N_{\mathrm{Ly} \alpha}=5 \times 10^{48} \mathrm{~s}^{-1}$ (Martins et al.

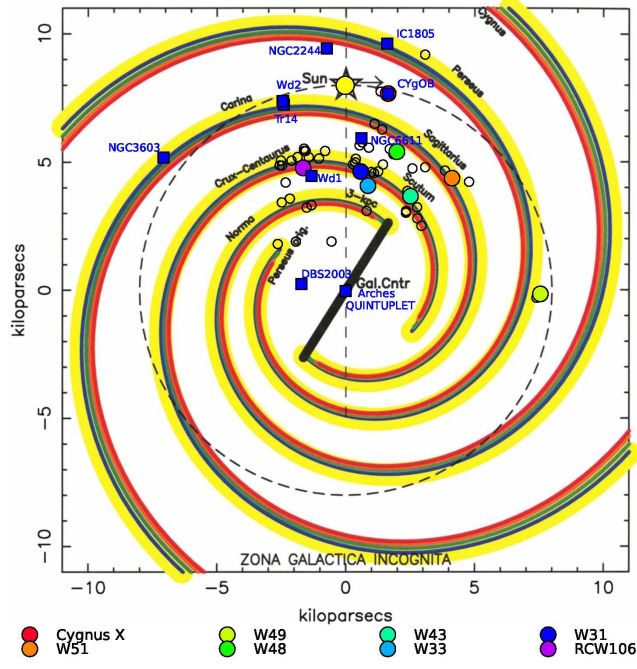

Figure 2. Location of the mini-starburst MCCs over the spiralarm structure of the Milky Way as modelled by Vallée (2014). Mini-starburst MCCs are indicated by circles. Well-known MCCs are labelled. Young Massive Clusters and OB Asscotiation from Portegies Zwart et al. (2010) are marked as squares, respectively.

2005), we calculate the SFR of the MCCs based on the SFR calibration for a full typical mass spectrum derived by (Murray \& Rahman 2010) as

$$
\frac{\mathrm{SFR}}{\mathrm{M}_{\odot} \mathrm{yr}^{-1}}=4.1 \times 10^{-54} \frac{N_{\mathrm{Ly} \alpha}}{\mathrm{s}^{-1}} .
$$

The SFR is then calculated for the entire MCCs using the area derived from $\mathrm{CO}$ emission (see Table 1 ). The SFR density of our MCCs range from 1 to 10 $\mathrm{M}_{\odot} \mathrm{yr}^{-1} \mathrm{kpc}^{-2}$, which are in the high range of the Gould Belt dense cores (Heiderman et al. 2010), although the sizes of MCCs are hundreds times as large. SFR densities of MCCs are comparable with the SFR of super giant $\mathrm{H}$ II regions in M33 (Miura et al. 2014). The fact that these high SFR densities fill up the missing part in the SFRMass diagram derived by Lada et al. (2010) suggests that MCCs are good candidates to link the SFRs from local GMC scale to global galaxy scale (see Figure 7).

When using the radio continuum flux to estimate the SFR of MCCs, a few assumptions have to be made, such as the mass spectrum over which the total stellar mass is calculated, the maximum cut-off mass of the mass spectrum, the non-dispersal property of the gas clouds, or the independence of the mass spectrum on the total gas mass. These assumption might add up uncertainties to our SFR measurements.

\section{GLOBAL LARSON'S SCALING RELATIONS AND BREAK AT MCC SCALES}

We analyse the scaling relations between different physical properties (mass, radius, gas mass density, and velocity dispersion) of cloud structures across 8 orders of magnitude in size and 13 orders of magnitude in mass using the most complete compilation of molecular cloud structures discussed in Section 2.3. To investigate the scale dependency of the scaling relations, we divide the dataset into three populations: $\operatorname{GMCs}(R<10 \mathrm{pc})$, 


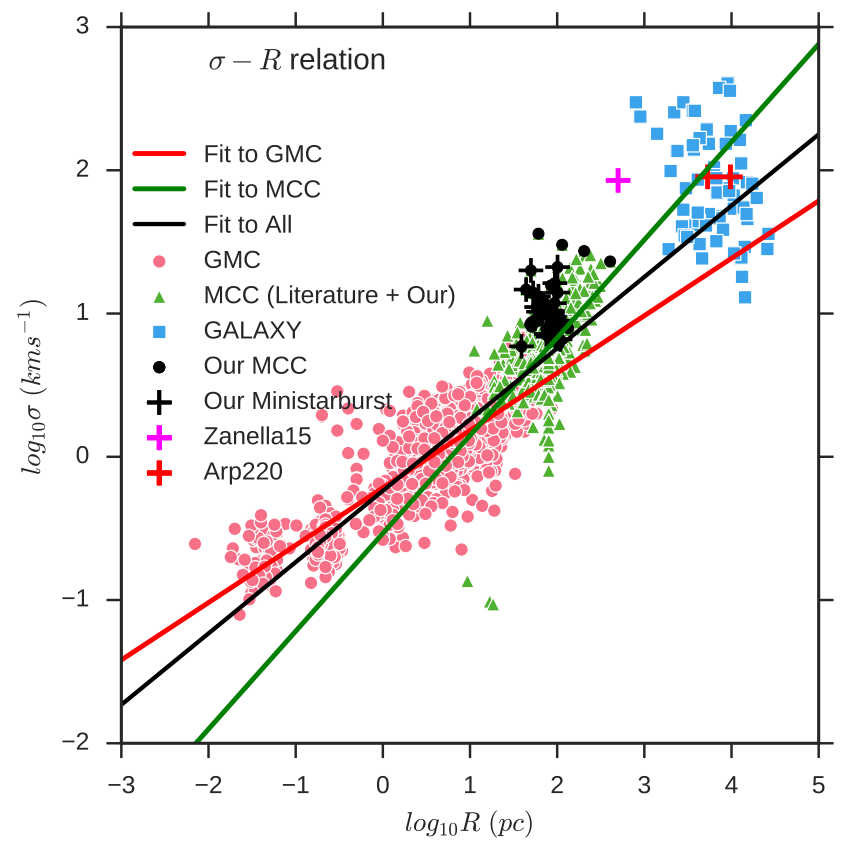

Figure 3. The Larson's scaling relation of velocity dispersionradius. All objects are divided into three categories: GMCs (size $<10 \mathrm{pc})$, MCC $(10<$ size $<1000 \mathrm{pc})$, and Galaxy (size $>1000 \mathrm{pc}$ ). The continuous lines are linear fits to the velocity dispersion-radius relations of GMC (pink), MCC (green), and all (black).

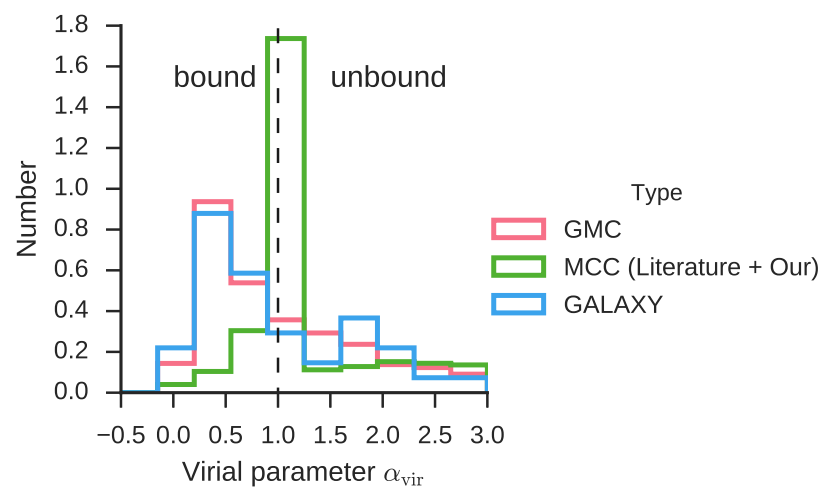

Figure 4. The histogram of the virial parameters $\alpha_{\text {vir }}$ of all objects which are divided into three categories: GMCs (size $<10$ pc), MCC $(10<$ size $<1000 \mathrm{pc})$, and Galaxy (size $>1000 \mathrm{pc})$. The dotted line divides the gravitaionally bound $\left(\alpha_{\mathrm{vir}}<1\right)$ and unbound $\left(\alpha_{\text {vir }}>1\right)$ regimes.

MCCs $(10<R<100 \mathrm{pc})$, galaxies $(R>100 \mathrm{pc})$. These sub-divisions contain literature data and our MCCs data. We then plot the velocity dispersion versus radius diagram to describe the first Larson's relation (Figure 3), the histogram of virial parameters to describe the second Larson's relation (Figure 4), the mass versus radius to present the third Larson's relation (Figure 5). Subsequently, we fit linear functions in log-log space to the $\sigma-R$ and $M-R$ to derive their power-laws relations.

As an independent check of the correlation between two parameters, we also calculate the Pearson correlation coefficient $r_{\mathrm{p}}$, which is the covariance of the two parameters divided by the product of their standard deviation. An $r_{\mathrm{p}}>0.7$ describes a strong correlated parameter pair, an $<0.3<r_{\mathrm{p}}<0.7$ describes a moderate correlated parameter pair, and an $r_{\mathrm{p}}<0.3$ describes an uncorrelated

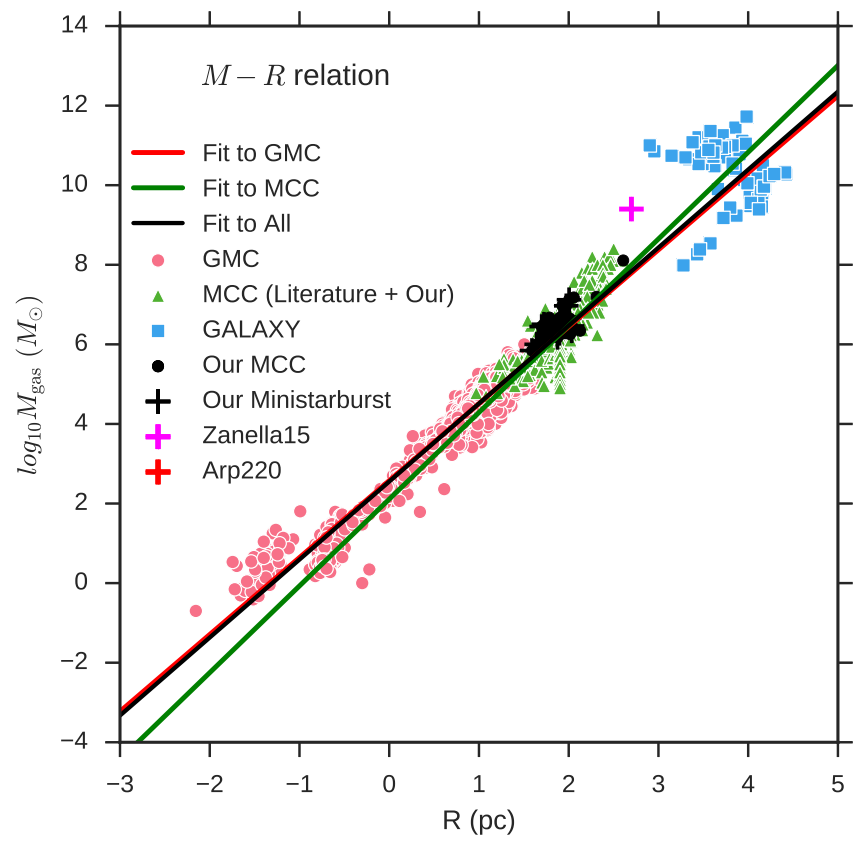

Figure 5. The Larson's scaling relation of mass-radius. Symbols are as in Figure 3. All objects are divided into three categories: GMCs (size $<10$ pc), MCC $(10<$ size $<1000$ pc), and Galaxy (size $>1000 \mathrm{pc}$ ). The continuous lines are linear fits to the mass-radius relations of GMC (pink), MCC (green), and all (black).

parameter pair. The positive sign indicates the positive correlation and vice versa.

\subsection{The $\sigma-R$ relation}

For the line width-size relation (Figure 3), we obtain the following results:

$$
\begin{aligned}
\text { GMC }: \sigma & =10^{-0.2} R^{0.4}, r_{p}=0.9 \\
\text { MCC }: \sigma & =10^{-0.6} R^{0.7}, r_{p}=0.6 \\
\text { All }: \sigma & =10^{-0.2} R^{0.5}, r_{p}=0.9
\end{aligned}
$$

The slope 0.4 of the power law fitted to GMCs is close to $\beta \sim 0.38$ measured by Larson (1981). This is in between the range of the slopes of the pure Kolmogorov turbulent structure $\left(\sigma \propto R^{0.33}\right)$ and the Burger shockgenerated one $\left(\sigma \propto R^{0.5}\right)$ cases. Simulations of cloud formation through turbulent shocks, for examples those of Bonnell et al. (2006) or Dobbs \& Bonnell (2007), also produce a $\sigma \propto R^{0.4}$ relation. However, the slope 0.7 of the fit to the MCC population is much higher than the GMC's one, even higher than the slope of the Burger shock-generated fractals.

As for the Pearson correlation coefficient, while the GMC and entire populations produce a good correlation between $\sigma$ and $R\left(r_{\mathrm{p}}=0.9\right.$ and $r_{\mathrm{p}}=0.9$, respectively), the MCC population has weaker correlation $\left(r_{\mathrm{p}}=0.6\right)$ and the galaxy population has no correlation $\left(r_{\mathrm{p}}=-0.2\right)$. Similarly, no relation between line-width and size is found for MCCs in nearby galaxies (Hughes et al. 2013; Utomo et al. 2015; Rebolledo et al. 2015). Together with our MCC data, we suggest that there is a break at the MCCs scale in addition to an apparent universal $\sigma-R$ relation. However, this break may be 
artificial due to different measurements such as tracers, methods, resolution and thresholds for the mass determination were used, or due to the intrinsic different properties of the samples. A more homogeneous investigation is therefore needed to confirm this break.

\subsection{The virial parameter histogram}

The second Larson relation describes the tendency of the molecular clouds to reach the virial equilibrium state. We check this hypothesis by examing the virial parameter $\sigma=5 \sigma_{1 \mathrm{D}}^{2} R / G M$. A cloud structure is dominated by gravitational energy if $\sigma \leq 1$, otherwise external pressure takes control. In Figure 4, we plot the histogram of virial parameters for different populations. The mean virial parameter of the GMCs is 1.0, of MCCs is 1.8, and of the entire sample is 1.2. Since $\sigma$ of GMCs is closer to 1 , the internal gravitational energy is stronger than kinetic energy as oppose to MCCs which are dominated by kinetic energy as their $\sigma$ is larger than 1 . In other words, GMCs are closer to be gravitationally bound than MCCs which are super virial and unbound. Similar results were found for MCCs in other galaxies (Hughes et al. 2013). For the case of our MCCs listed in Table 1, their virial parameters are even larger. This picture is also seen in simulations where most of the large structures are unbound and the small structure are bound (Dobbs et al. 2011; Fujimoto et al. 2014). The large velocity dispersion may be caused by the fact that MCC is a more dynamic system that can form a compressive environment. Susequently, this results in a higher specific star formation rate or star formation density as we discussed later on.

$$
\text { 5.3. The } M_{\text {gas }}-R \text { relation }
$$

The third Larson relation implies that the mass and size obey a power relation $M_{\text {gas }} \propto R^{\alpha}$ with $\alpha \sim 2$, or all molecular cloud structures have similar mass surface density, which we examine in Figure 5. The best fits to the GMCs, MCCs, galaxies and entire samples yield slopes of $1.9,2.2$, and 2.0, respectively:

$$
\begin{aligned}
& \mathrm{GMC}: M_{\text {gas }}=10^{2.6} R^{1.9}, r_{p}=1.0 \\
& \mathrm{MCC}: M_{\text {gas }}=10^{2.1} R^{2.2}, r_{p}=0.7 \\
& \text { All }: M_{\text {gas }}=10^{2.6} R^{2.0}, r_{p}=1.0
\end{aligned}
$$

All three slopes are very close to $M_{\text {gas }} \propto R^{2}$ although that of MCCs slightly deviates from 2. They are in between the value of $\sim 1.6$ for substructures within individual clouds (Lombardi et al. 2010; Kauffmann et al. 2010) and the value of $\sim 2.4$ derived for GMCs in the Galactic Plane GRS survey (Roman-Duval et al. 2010). The good correlation between mass and size are also present in the large Pearson coefficients: 1.0 for GMCs, 0.7 for MCCs, and 1.0 for the entire population.

The result of this Section is that there is an apparent universal relation between velocity dispersion and size, mass and size of cloud structures across 8 orders of magnitude in size and 13 orders of magnitude in mass. However, there is a break at the MCC scale in the $\sigma-R$ relation and the slopes of individual populations are slightly different. The virial parameters of GMCs and Galaxies are close to 1 while those of MCCs are larger than 1 , thus signifies the importance of the kinetic energy contribution in regulating the MCC structures.

\section{THE STAR FORMATION RATES - GAS PROPERTIES RELATION}

Similar as in Section 5, we construct the scaling relations and their best-fitted power law models between the $S F R$ surface density $\Sigma_{\text {SFR }}$ and mass surface density $\Sigma_{\text {gas }}$, between the SFR and the total mass $M_{\text {gas }}$. In addition, we also calculate the Pearson correlation coefficients for different pairs of parameters.

\subsection{The Schmidt-Kennicutt $\Sigma_{\mathrm{SFR}}-\Sigma_{M_{\text {gas }}}$ relation}

The $\Sigma_{\text {SFR }}-\Sigma_{M_{\text {gas }}}$ relation was first constructed by Kennicutt (1998) for the integrated SFR density $\Sigma_{\text {SFR }}$ and the total gas mass density $\Sigma_{M_{\text {gas }}}$ of normal starforming and starburst galaxies. Since then, it was generated as one of the most applicable tool to explain the universal role of gravity in forming stars. Recently, the relation has been extended to GMCs scale (Heiderman et al. 2010; Krumholz et al. 2012b) in an endeavour to establish a universal star formation law that connects local to global scales. We re-investigate this relation for our combined dataset by constructing the SFR density and gas mass density relation in Figure 6 and deriving their best linear fits to the $\Sigma_{\mathrm{SFR}}-\sigma$ relations in the loglog space as:

$$
\begin{array}{r}
\text { GMC : } \Sigma_{\mathrm{SFR}}=10^{-4.9} \Sigma_{\mathrm{M}_{\mathrm{gas}}}{ }^{2.4}, r_{p}=0.8 \\
\text { MCC : } \Sigma_{\mathrm{SFR}}=10^{-3.0} \Sigma_{\mathrm{M}_{\mathrm{gas}}}{ }^{1.3}, r_{p}=0.5 \\
\text { GALAXY : } \Sigma_{\mathrm{SFR}}=10^{-3.8} \Sigma_{\mathrm{M}_{\mathrm{gas}}}{ }^{1.4}, r_{p}=1.0 \\
\text { ALL : } \Sigma_{\mathrm{SFR}}=10^{-3.4} \Sigma_{\mathrm{M}_{\mathrm{gas}}} 1.5, r_{p}=0.7
\end{array}
$$

The slopes of the $\Sigma_{\mathrm{SFR}}-\Sigma_{M_{\mathrm{gas}}}$ relations are different among different populations. While GMCs have the steepest slope of 2.4, MCCs have the most shallow slope of 1.3, Galaxies have a slope that is closest to the original value of 1.4 derived by Kennicutt (1998). The steeper slope of the GMC population is consistent with that of the star-forming clump population (Heiderman et al. 2010) and of the resolved individual MCCs (Willis et al. 2015). From a global view of our data, we derive a universal Schmidt-Kennicutt scaling relation with a slope of 1.5 and large scatters in the GMC and MCC populations. The Pearson coefficients show that $\Sigma_{\mathrm{SFR}}$ and $\Sigma_{M_{\text {gas }}}$ correlate strongly $\left(r_{p}=0.9\right)$ in the galaxy population while they correlate least in the MCC population $\left(r_{p}=0.5\right)$.

\subsection{The $\mathrm{SFR}-M_{\text {gas }}$ relation}

Instead of comparing the surface density quantities of SFR and mass, we examine the integrated SFR and the integrated gas mass relation in Figure 7 . Our literature and new data of MCCs fill the $S F R-M_{\text {gas }}$ plane and connect the smallest cloud scale to galaxy scale. We plot in Figure 7 the relation between SFR and $M_{\text {gas }}$ in the log-log space and fits linear functions to them to obtain the following results for different populations:

$$
\begin{aligned}
& \text { GMC }: \mathrm{SFR}=10^{-7.4} \mathrm{M}_{\mathrm{gas}}{ }^{0.8}, r_{p}=0.7 \\
& \mathrm{MCC}: \mathrm{SFR}=10^{-6.2} \mathrm{M}_{\mathrm{gas}}{ }^{0.6}, r_{p}=0.4 \\
& \text { GALAXY : } \mathrm{SFR}=10^{-11.7} \mathrm{M}_{\mathrm{gas}}{ }^{1.3}, r_{p}=0.9 \\
& \text { ALL : } \mathrm{SFR}=10^{-7.6} \mathrm{M}_{\mathrm{gas}}{ }^{0.9}, r_{p}=1.0 \text {. }
\end{aligned}
$$



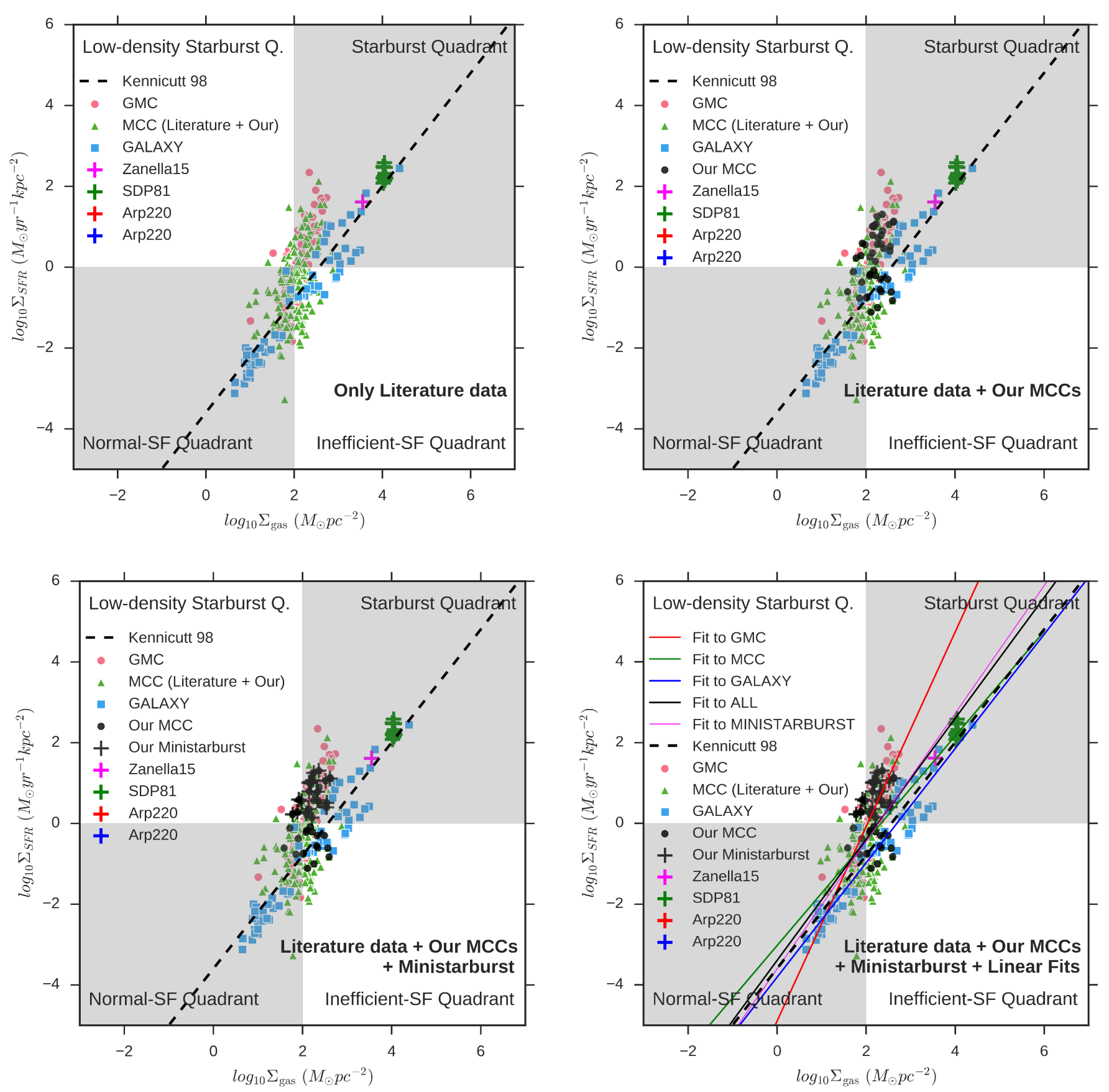

Figure 6. The Schmidt-Kennicutt relation of $\Sigma_{\mathrm{SFR}}-\Sigma_{M_{\text {gas }}}$ for objects ranging from Milky Way clouds to unresolved galaxies and the best fits. The white and grey regions are divided by the gas surface density of $100 \mathrm{M}_{\odot} \mathrm{pc}^{-2}$ and the SFR density of $1 \mathrm{M}_{\odot} \mathrm{yr}^{-1} \mathrm{kpc}^{-2}$. Top-Left diagram includes only literature data, Top-Right diagram includes literature data and our MCCs from this study, Bottom-Left diagram includes literature data, our MCCs and mini-starburst MCCs from this study, and Top-Right diagram includes all data and different linear fits as discussed in Equations 10-13 in Section 6.1. We note that the dense cores samples $(R<1 \mathrm{pc})$ from Maruta et al. $(2010)$, Onishi et al. (2002), and Shimajiri et al. (2015) are not included in the SFR analysis due to the lack of SFR measurements. 
The slopes of our fits are super-linear for the Galaxy population and sub-linear for all other cases. The slope of the fit to the combined data, is sub-linear with a slope of 0.87 , which does not quite agree with the unity slopes derived by $\mathrm{Wu}$ et al. (2005) or Lada et al. (2010). Thus to the first order, this universal scaling relation is useful to establish a common relation between SFR and the total gas mass for all star-forming objects. However, at the individual scales, this relation has strong scatter and the linear fits to the data vary strongly. The strong correlation between SFR and $M_{\text {gas }}$ of the entire dataset can also be seen in the large Pearson coefficient $\left(r_{p}=0.95\right)$. Similar as in the $\Sigma_{\mathrm{SFR}}-\Sigma_{\text {gas }}$ relation, the MCC has very low Pearson coefficient $\left(r_{p}=0.37\right)$, indicating the low correlation between $S F R$ and $M_{\text {gas }}$. For example, the total SFR of MCCs vary almost over 4 orders of magnitude. However, they are consistent with the recent numerical simulation of star formation activities in MCCs, these simulation show large SFR spreads in the $S F R-M_{\text {gas }}$ diagram (Howard et al. 2016). Lada et al. (2012) suggested that the spread in $S F R-M$ diagram is the effect of the dense gas fraction which we could not address with the current data. While we would expect that the slopes of the $S F R-M$ relations are super-linear as similar as those of $\Sigma_{\mathrm{SFR}}-\Sigma_{M_{\text {gas }}}$ relations, they are however sub-linear. The reasons is that the gas surface density also scales with Area as $A \sim \Sigma_{M_{\text {gas }}}^{-q}$. Therefore, if there is $S F R \propto M_{\text {gas }}^{p}$, we should get $\Sigma_{\mathrm{SFR}} \propto A^{-1} \Sigma_{M_{\mathrm{gas}}}^{p}=A^{p-1} \Sigma_{M_{\mathrm{gas}}}^{p}$. The dependency of gas surface density on area has been investigated by Burkert \& Hartmann (2013), who showed that $\Sigma_{M_{\text {gas }}}$ scales with Area as $A \sim \Sigma_{M_{\text {gas }}}^{-3}$ for low-mass star forming regions and $A \sim \Sigma_{M_{\text {gas }}}^{-1}$ for massive cores .

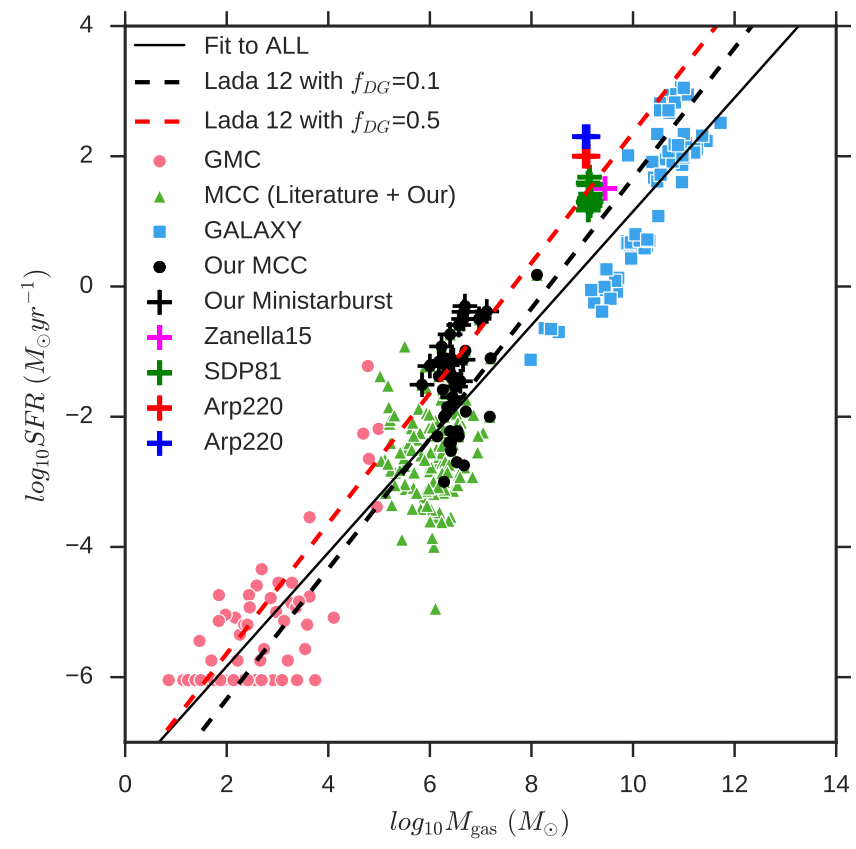

Figure 7. The SFR $-M_{\text {gas }}$ relation for objects ranging from Milky Way clouds to unresolved galaxies and the best fits.

\subsection{The $\mathrm{SFR}-\sigma$ relation}

Ideally, if all of the scaling relations in Section 5.1, 5.3, and 6.2 hold together, we can deduce a relation between $\Sigma_{\mathrm{SFR}}-\sigma$ from the other relations. Because $\sigma \propto R^{0.3-0.5}$ and $\mathrm{M}_{\text {gas }} \propto R^{2}$, we have the relation $\mathrm{M}_{\text {gas }} \propto \sigma^{4-7}$. As a consequence of the relation $\mathrm{SFR} \propto \mathrm{M}_{\text {gas }}{ }^{(0.8-1.3)}$, we should have a relation $\mathrm{SFR} \propto \sigma^{(3.1-8.8)}$. However, as shown in Figure 8, we obtain different slopes from fitting power laws to the individual populations and also combined dataset:

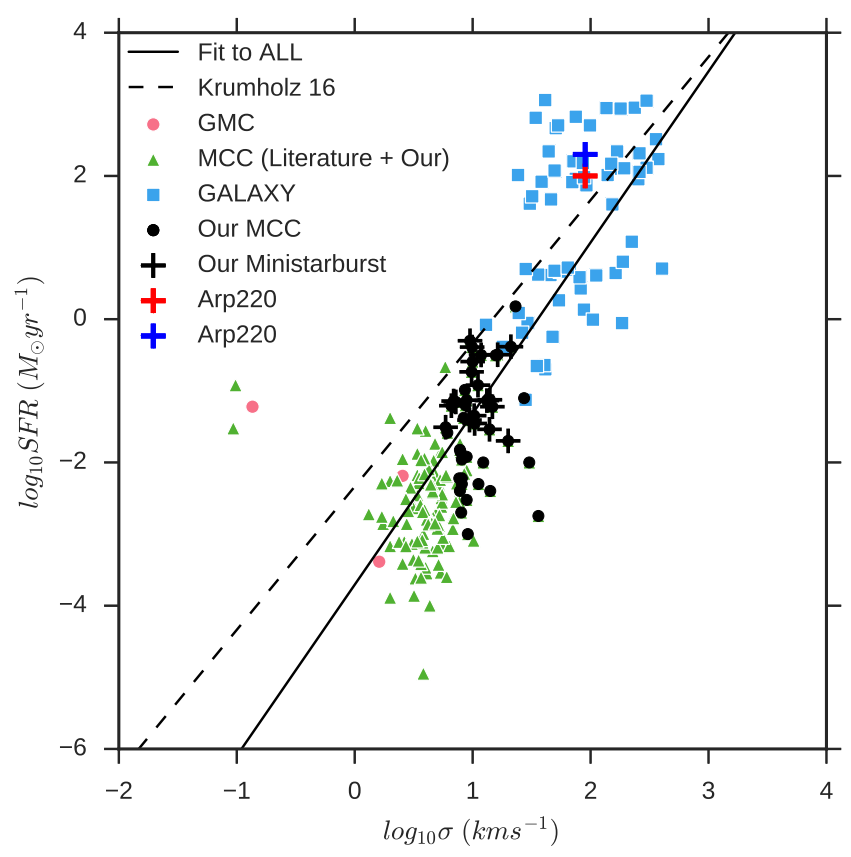

Figure 8. The SFR $-\sigma$ (SFR - velocity dispersion) relation for objects ranging from Milky Way clouds to unresolved galaxies and their best fits. There are less GMC data points because there are less GMCs that have both SFR and velocity dispersion measurements.

$$
\begin{array}{r}
\text { MCC : } \mathrm{SFR}=10^{-2.9} \sigma^{0.9}, r_{p}=0.3 \\
\text { GALAXY : } \mathrm{SFR}=10^{-1.8} \sigma^{1.9}, r_{p}=0.8 \\
\text { ALL : } \mathrm{SFR}=10^{-3.8} \sigma^{2.7}, r_{p}=0.8
\end{array}
$$

The empirical fits show that SFR increases with the velocity dispersion but the slopes are much shallower than the theoretical prediction from other scaling laws. A global fit with a slope of 2.7 fits well to the entire dataset. But fits to GMC and MCC populations are uncertain because their velocity dispersion ranges are less than an order of magnitude.

Krumholz \& Burkhart (2016) created feedback-driven models and gravity-driven models to explain the SFR- $\sigma$ relations in the galaxy interstellar media and found that the former model produces a steeper relation SFR $-\sigma^{2}$ while the later model produces a shallower and dense-gas-mass-fraction dependence relation $\mathrm{SFR}-f_{\mathrm{DG}} \sigma$. These slopes are close to the slope of our galaxy population data, however, they are not applicable to MCCs and GMCs, which seem to be fitted with 
steeper slopes. Similarly at the previous cases, MCCs have smallest Pearson coefficient $\left(r_{p}=0.3\right)$ or their $S F R$ and $\sigma$ are not correlated well.

\section{DISCUSSION \& CONCLUSION}

\subsection{Four quadrants of the Schmidt-Kennicutt diagram}

One of the application of the $\Sigma_{\mathrm{SFR}}-\Sigma_{\text {gas }}$ diagram is to distinguish the starburst from the normal galaxies. Daddi et al. (2010), for example, argued that the two different regimes of star formation in galaxies are caused by the longer depletion time of normal galaxies than that of the starburst. For the same gas surface density, the starburst galaxies and mini-starburst MCCs have higher SFR density than the normal star forming galaxies and normal star-forming MCCs, therefore lie at a higher location in the Schmidt-Kennicutt diagram. However, the scatter in the $\Sigma_{\text {SFR }}-\Sigma_{\text {gas }}$ are getting larger as there are better observations, both in galactic and extragalactic scales, which make the recognition of small deviation from the universal law unnoticeable. Using our combined data, we propose an alternative way to distinguish starburst from normal star-forming objects by applying the $\Sigma_{\mathrm{SFR}}$ and $\sigma$ thresholds to the Schmidt-Kennicutt diagram.

A $\Sigma_{\text {gas }}$ threshold of $\sim 100-120 \mathrm{M}_{\odot} \mathrm{pc}^{-2}$ was suggested as the borderline between star-forming and non star-forming clouds or between normal spiral galaxies and starburst galaxies (Lada et al. 2010; Heiderman et al. 2010). As we can already see in the SchmidtKennicutt diagram in Figure 6, som MCCs or galaxies, and even some GMCs have star formation at $\Sigma_{\text {gas }}<$ $100 \mathrm{M}_{\odot} \mathrm{pc}^{-2}$. However, this threshold can be safely used as a threshold dividing different star formation modes: isolated versus clustered, normal versus starburst, while the later ones always require more gas with $\Sigma_{\text {gas }}>100-120 \mathrm{M}_{\odot} \mathrm{pc}^{-2}$ (Wu et al. 2005; Kennicutt 1998). There is exception such as the central molecular zone in the Milky Way, which has high gas mass surface density but low SFR density. Additionally, we use the $\Sigma_{\mathrm{SFR}} \sim 1 \mathrm{M}_{\odot} \mathrm{yr}^{-1} \mathrm{kpc}^{-2}$ as the threshold between starburst and non-starburst objects. This SFR threshold was originally used to distinguish between starburst and normal-star forming galaxies (Kennicutt 1998).

Putting these two thresholds into the SchmidtKennicut diagram, as in Figure 6, the four quadrants are divided and named clockwise as: low-density starburst quadrant, normal star-forming quadrant, inefficient-star forming quadrant, and starburst quadrant. Structures in the starburst quadrant have high gas density and high star formation efficiency such as starburst galaxy and ministarburst complex. Structures in the inefficient starformation quadrant also have high gas density, however their star formation is impeded. The Central Molecular Zone is a good example of the exception case (Immer et al. 2012). The majority of structures in the normal-star formation quadrant are galaxies and they follow well the Schmitt-Kennicutt fit with much less scatter than objects in the starburst quadrant. In the lowdensity starburst quadrant, the low gas density inhibit high SFR density higher than $1 \mathrm{M}_{\odot} \mathrm{yr}^{-1} \mathrm{kpc}^{-2}$. Nevertheless, there are maybe a few candidates that have $\Sigma_{\mathrm{SFR}}>1 \mathrm{M}_{\odot} \mathrm{yr}^{-1} \mathrm{kpc}^{-2}$, or they might be the outliers.

\subsection{Definition of mini-starburst complexes}

The starburst phenomenon was first suggested by observations of the excess star formation activity in galaxy nuclei (Arp \& O’Connell 1975; Huchra 1977). More recently, Elbaz et al. (2011) proposed that a galaxy experiences a starburst phase if its 'current SFR' is twice higher than its SFR averaged over time and used to define its 'main sequence SFR'. With a total gas mass of $1 \times 10^{9} \mathrm{M}_{\odot}$ (Dame et al. 1993), the Milky Way becomes a starburst galaxy only if its SFR is as high as 20 times of the current rate of $\sim 0.7-2 \mathrm{M}_{\odot} \mathrm{yr}^{-1}$ (Robitaille \& Whitney 2010). The Milky Way as a whole, is therefore far from experiencing a starburst phase. However, SFR is not uniformly distributed across the Milky Way but excess star formation activity exist in MCC that forms massive star clusters (Nguyen Luong et al. 2011a; Murray 2011).

We define the mini-starburst MCCs as objects having these properties:

- total gas mass is larger than $10^{6} \mathrm{M}_{\odot}$,

- gravitationally unbound,

- Star formation rate density is larger than $1 \mathrm{M}_{\odot} \mathrm{yr}^{-1} \mathrm{kpc}^{-2}$ or its location is on the starburst quadrant in the Schmidt-Kennicutt diagram (see Figure 6).

From the 44 massive MCCs detected in Section 3, we obtain 21 mini-starburst MCCs. Most of them are the famous mini-starburt MCCs studied extensively in the literatures, for example:

- $R C W 106$ is the second brightest MCC in our survey, resides in the Scutum-Centaurus arm and surrounds the bright giant $\mathrm{H}$ II region RCW 106 hosting a rich OB cluster (Rodgers et al. 1960; Nguyen et al. 2015).

- W43 lies at the meeting point of the ScutumCentaurus (or Scutum-Crux) arm and the Bar. This mini-starburst is a prototypical example of a mini-starburst MCC (Nguyen Luong et al. 2011b; Carlhoff et al. 2013).

- W49 lies on the Perseus arm and hosts ongoing starburst event and forms a very massive star with mass from 100-190 M $\odot$ (Galván-Madrid et al. 2013)

- Cygnus $X$ is one of the most massive mini-starburst MCC and is located in the Cygnus arm (Schneider et al. 2006).

- W51 is near the tangent point of the Sagittarius arm, has a high dense gas fraction and host massive star formation events (Ginsburg et al. 2015).

We compare the Galactic mini-starburst with the extragalactic mini-starburst which are resolved to a comparable scale. First, we use data from Arp 220, a relatively nearby $(\mathrm{d} \sim 75 \mathrm{Mpc})$ ultraluminous infrared galaxy (Soifer et al. 1984), which contains two nuclei that are powered by extreme starburst activity. Sakamoto et al. (2008); Wilson et al. (2014); Scoville et al. (2015) 
used ALMA to resolve the Arp 220 down to a spatial scale of $\sim 100 \mathrm{pc}$ and obtain gas mass densities of $5.4 \times 10^{4}$ and $14 \times 10^{4} \mathrm{M}_{\odot} \mathrm{pc}^{-2}$ for the Eastern and Western nucleus. Taking into account the SFR of $100-200 \mathrm{M}_{\odot} \mathrm{yr}^{-1}$ (Scoville et al. 2015), we obtain a SFR density $10^{4}-10^{4.5} \mathrm{M}_{\odot} \mathrm{yr}^{-1} \mathrm{kpc}^{-2}$, the highest SFR density at the MCC scale. Second, we use data of 14 starburst MCC clumps in SDP.81 galaxy derived from ALMA observations. SDP.81 is one of the bright galaxy at a redshift $z=3.042$ (or a luminosity distance of $\sim 25 \times 10^{3} \mathrm{Mpc}$ ) and is gravitationally lensed by a foreground galaxy at $\mathrm{z}=0.2999$, therefore it allows us to resolve the gas properties down the scale of $\sim 200$ pc by ALMA (Hatsukade et al. 2015). Finally, we also include in our comparison the data from the first direct measurement of a MCC clump at $\mathrm{z}=1.987$ down to the scale of $\sim 500$ pc (Zanella et al. 2015). Although they are larger than our mini-starburst, we still compare them with our data keeping in mind that their $\sigma$ and $\Sigma_{\mathrm{SFR}}$ can be higher if we resolve them at a smaller scale.

The SFR and SFR density of the extragalactic ministarburst are ten to hundred times higher than the Galactic counterparts that they are located in the upper part of the starburst quadrant and the upper part of the SFR $-M_{\text {gas }}$ diagram. Highly-compressed gas in these extragalactic mini-starbursts may be the origin of their active star formation activities.

\subsection{Dynamical Evolution of mini-starburst MCCs}

We advocate that the high $\Sigma_{\mathrm{SFR}}$ and high $\sigma$ of a mini-starburst MCC is caused by dynamical processes happen during the MCCs evolution. These processes are supported by externally induced pressure such as shocks, galactic disk gravitational instability or colliding flow (Vazquez-Semadeni et al. 1996; Bonnell et al. 2013). Therefore, mini-starburst MCCs are often found at highly dynamics regions such as the overlapping regions in Antennae galaxy (Herrera et al. 2012; Fukui et al. 2014) or at the end of the Galactic Bar (Nguyen Luong et al. 2011b). Simulation of molecular cloud evolution in a galaxy also agrees with this view by showing that MCCs are concentrated mostly in the Bar or spiral arms, and have high SFR (Fujimoto et al. 2014).

In all cases, continuous gas flows agglomerate clouds, compress material and develop active star formation sites (e.g., Koyama \& Inutsuka 2000; Bergin et al. 2004), which explains why mini-starburst MCCs have high SFR (see Sect. 7.2). This framework is also called cloud-cloud collision, advocated as the main formation mechanism of massive star and stellar cluster (Inoue \& Fukui 2013). For example, in W43, one of the most active massive star-forming regions, both large-scale and small-scale gas flows were observed as a mean of forming dense gas and massive stars (Motte et al. 2014; Nguyen-Luong et al. 2013; Louvet et al. 2014).

In addition, a super-linear relation between $S F R-\sigma$ (Figure 8) indicating that SFR increases with the turbulence or compression degrees of the gas supports the dynamical view of MCC evolution. The steep slope of the $S F R-\sigma$ relation, especially that of MCC, disagrees with the slope produced by the gravity-driven models or feedback-driven model (Krumholz \& Burkhart 2016). Therefore, cloud compression plays a stronger role in

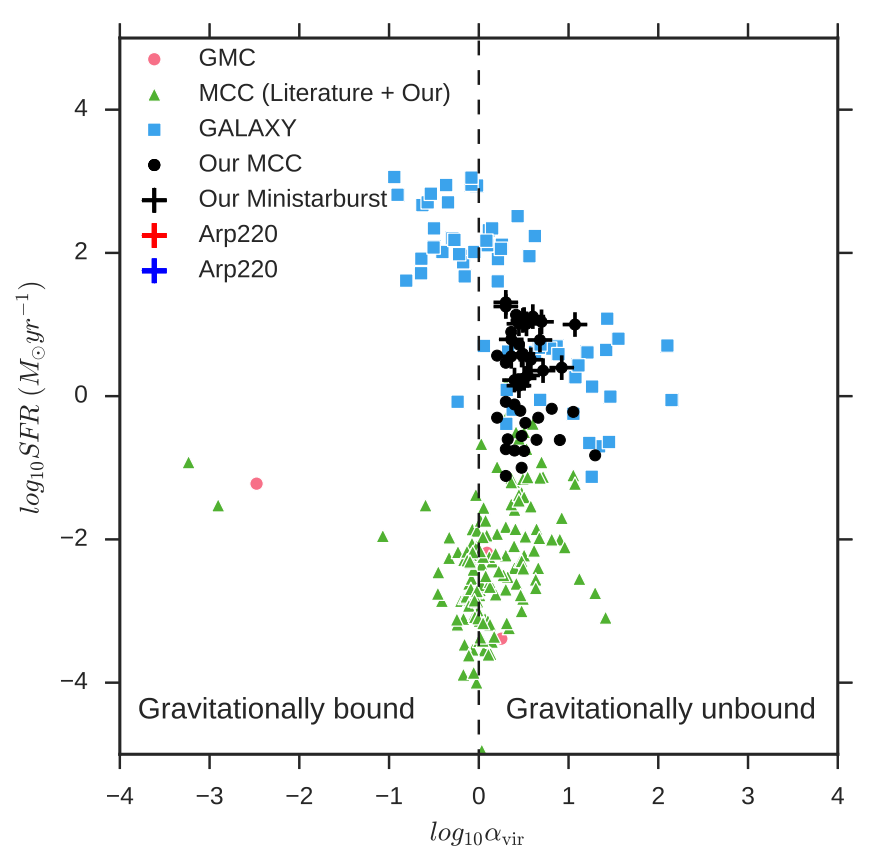

Figure 9. The SFR $-\alpha_{\text {vir }}$ (SFR - virial parameter) relation for objects ranging from Milky Way clouds to unresolved galaxies. It shows that there is no particular relation between SFR and $\sigma$ but the mini-starburst MCCs are mostly gravitationally unbound. There are less GMC data points because there are less GMCs that have both SFR and velocity dispersion measurements.

controlling the structure and the star formation activity, beyond gravity and feedback. As a consequence, the majority of MCCs are gravitational unbound and form stars efficiently as shown in Figure 9, especially th ministarburst MCCs.

\section{CONCLUSIONS}

We investigated the connection between the local and global star formation by comparing the mass, size, line width, and star formation rate of cloud structures across 8 orders of magnitude in size and 13 orders of magnitude in mass. Our focus is on molecular cloud complexes (MCCs), which have radii of $\sim 50-70 \mathrm{pc}$ and masses $>10^{6} \mathrm{M}_{\odot}$. We use the ${ }^{12} \mathrm{CO} 1-0 \mathrm{CfA}$ survey to identify and characterize a sample of 44 MCCs in the Milky Way (see Table 1). This sample is complete up to a distance of $6 \mathrm{kpc}$ from the Sun. Their distribution follows the spiral arms, especially the Scutum-Centaurus and Sagittarius arms (see Figures 1-2).

Together with data from the literature, we reproduced the scaling relations and the star formation laws: $\sigma-R$, $M_{\text {gas }}-R, \Sigma_{\mathrm{SFR}}-\Sigma_{M_{\text {gas }}}, \mathrm{SFR}-M_{\text {gas }}$, and $\mathrm{SFR}-\sigma$. Apart from being apparently universal, the slopes and the coefficients are different for individual scales: GMC, MCC, and galaxy. Second, there is a break at the MCC scale in the $\sigma-R$ relation and a break between the starburst objects such as mini-starburst, star-forming clumps from the normal star-forming objects in the SFR- $M_{\text {gas }}$ and $\Sigma_{\mathrm{SFR}}-\Sigma_{M_{\text {gas }}}$ relations.

These breaks enable us using the Schmidt-Kennicutt diagram to distinguish the starburst from the normal star-forming objects by using the $\Sigma_{M_{\text {gas }}}$ threshold of $100 \mathrm{M}_{\odot} \mathrm{pc}^{-2}$ and the $\Sigma_{\mathrm{SFR}}$ threshold of $1 \mathrm{M}_{\odot} \mathrm{yr}^{-1}$ 
$\mathrm{kpc}^{-2}$. These two thresholds divide the $\Sigma_{\mathrm{SFR}}-\Sigma_{M_{\mathrm{gas}}}$ diagram into four quadrants: Q1 as low-density starburst quadrant, Q2 as normal star-forming quadrant, Q3 as inefficient-star forming quadrant, and Q4 as starburst quadrant. Mini-starburt MCC are gravitationally unbound MCCs that have enhanced SFR density that is larger than $1 \mathrm{M}_{\odot} \mathrm{yr}^{-1} \mathrm{kpc}^{-2}$.

We propose that mini-starburst MCC is formed through a dynamical process, which enhance the compression of clouds and induce intense star formation as bursts and eventually form young massive star cluster. Because of the dynamical evolution, gravitational boundedness does not play a significant role in characterizing the star formation activity of mini-starburst MCCs. Therefore, there is no particular relation between SFR and the virial parameter (see Figures 8-9).

QNL acknowledges the financial support from the East Asian Core Observatories Association (EACOA) through the EACOA fellow program and the support from Canadian Institute for Theoretical Astrophysics during his visit at CITA. N.S. acknowledges support through the DFG project numbers 0s 177/2-1 and 177/2-2, and central funds of the DFG-priority program ISM-SPP. We thank the anonymous referee and Neal J. Evans for giving constructive comments which improve the quality of the paper.

\section{REFERENCES}

Anderson, L. D., \& Bania, T. M. 2009, ApJ, 690, 706 [1] Arp, H., \& O'Connell, R. W. 1975, ApJ, 197, 291 [7.2]

Barnes, P. J., Muller, E., Indermuehle, B., et al. 2015, ApJ, 812, 6 $[1,4.1]$

Bergin, E. A., Hartmann, L. W., Raymond, J. C., \& Ballesteros-Paredes, J. 2004, ApJ, 612, 921 [7.3]

Bigiel, F., Leroy, A., Walter, F., et al. 2008, AJ, 136, 2846 [1]

Blitz, L., \& Williams, J. P. 1999, in NATO ASIC Proc. 540: The Origin of Stars and Planetary Systems, ed. C. J. Lada \& N. D. Kylafis, 3-+ [1]

Bolatto, A. D., Leroy, A. K., Rosolowsky, E., Walter, F., \& Blitz, L. 2008, ApJ, 686, 948 [2.3]

Bolatto, A. D., Wolfire, M., \& Leroy, A. K. 2013, ARA\&A, 51, 207 [4.1]

Bonnell, I. A., Dobbs, C. L., Robitaille, T. P., \& Pringle, J. E. 2006, MNRAS, 365, 37 [5.1]

Bonnell, I. A., Dobbs, C. L., \& Smith, R. J. 2013, MNRAS, 782 [7.3]

Bressert, E., Ginsburg, A., Bally, J., et al. 2012, ApJ, 758, L28 [1]

Bronfman, L., Alvarez, H., Cohen, R. S., \& Thaddeus, P. 1989, ApJS, 71, 481 [2.1]

Burkert, A., \& Hartmann, L. 2013, ApJ, 773, 48 [6.2]

Carlhoff, P., Nguyen Luong, Q., Schilke, P., et al. 2013, A\&A, 560, A24 [3, 7.2]

Chibueze, J. O., Omodaka, T., Handa, T., et al. 2014, ApJ, 784, 114 [1]

Choi, Y. K., Hachisuka, K., Reid, M. J., et al. 2014, ApJ, 790, 99 [1]

Cohen, R. S., Cong, H., Dame, T. M., \& Thaddeus, P. 1980, ApJ, 239, L53 [1]

Daddi, E., Elbaz, D., Walter, F., et al. 2010, ApJ, 714, L118 [1, 7.1]

Dame, T. M., Elmegreen, B. G., Cohen, R. S., \& Thaddeus, P. 1986, ApJ, 305, 892 [1, 2.1]

Dame, T. M., Hartmann, D., \& Thaddeus, P. 2001, ApJ, 547, 792 $[1,2.1,3,4.1]$

Dame, T. M., Koper, E., Israel, F. P., \& Thaddeus, P. 1993, ApJ, 418, 730 [7.2]

Deharveng, J.-M., Sasseen, T. P., Buat, V., et al. 1994, A\&A, 289,715 [1]
Dempsey, J. T., Thomas, H. S., \& Currie, M. J. 2013, ApJS, 209, 8 [1]

Dobbs, C. L., \& Bonnell, I. A. 2007, MNRAS, 374, 1115 [5.1]

Dobbs, C. L., Bonnell, I. A., \& Pringle, J. E. 2006, MNRAS, 371, 1663 [4.1]

Dobbs, C. L., Burkert, A., \& Pringle, J. E. 2011, MNRAS, 413, 2935 [5.2]

Donovan Meyer, J., Koda, J., Momose, R., et al. 2013, ApJ, 772, $107 \quad[2.3]$

Elbaz, D., Dickinson, M., Hwang, H. S., et al. 2011, A\&A, 533, A119 [7.2]

Elmegreen, B. G., Morris, M., \& Elmegreen, D. M. 1980, ApJ, $240,455[1]$

Evans, II, N. J., Heiderman, A., \& Vutisalchavakul, N. 2014, ApJ, $782,114[1,2.3]$

Fujimoto, Y., Tasker, E. J., \& Habe, A. 2014, MNRAS, 445, L65 $[4.1,5.2,7.3]$

Fukui, Y., Ohama, A., Hanaoka, N., et al. 2014, ApJ, 780, 36 [7.3]

Galván-Madrid, R., Liu, H. B., Zhang, Z.-Y., et al. 2013, ApJ, $779,121[4.1,7.2]$

Gao, Y., \& Solomon, P. M. 2004, ApJS, 152, 63 [1]

García, P., Bronfman, L., Nyman, L.-Å., Dame, T. M., \& Luna, A. 2014, ApJS, 212, 2 [2.3, 4.1]

Genzel, R., Tacconi, L. J., Gracia-Carpio, J., et al. 2010, MNRAS, 407, 2091 [2.3]

Ginsburg, A., Bally, J., Battersby, C., et al. 2015, A\&A, 573, A106 [4.1, 7.2]

Hatsukade, B., Tamura, Y., Iono, D., et al. 2015, PASJ, 67, 93 $[2.3,7.2]$

Haverkorn, M., Gaensler, B. M., McClure-Griffiths, N. M., Dickey, J. M., \& Green, A. J. 2006, ApJS, 167, 230 [2.2]

Heiderman, A., Evans, II, N. J., Allen, L. E., Huard, T., \& Heyer, M. 2010, ApJ, 723, 1019 [1, 2.3, 4.2, 6.1, 6.1, 7.1]

Herrera, C. N., Boulanger, F., Nesvadba, N. P. H., \& Falgarone, E. 2012, A\&A, 538, L9 [1,7.3]

Heyer, M., Krawczyk, C., Duval, J., \& Jackson, J. M. 2009, ApJ, 699, $1092[1,2.3,4.1]$

Honma, M., Bushimata, T., Choi, Y. K., et al. 2007, PASJ, 59, 889 [3]

Howard, C. S., Pudritz, R. E., \& Harris, W. E. 2016, MNRAS, $461,2953[6.2]$

Huchra, J. P. 1977, ApJ, 217, 928 [7.2]

Hughes, A., Meidt, S. E., Colombo, D., et al. 2013, ApJ, 779, 46 $[1,5.1,5.2]$

Immer, K., Menten, K. M., Schuller, F., \& Lis, D. C. 2012, A\&A, 548, A120 [7.1]

Immer, K., Reid, M. J., Menten, K. M., Brunthaler, A., \& Dame, T. M. 2013, A\&A, 553, A117 [1]

Inoue, T., \& Fukui, Y. 2013, ApJ, 774, L31 [7.3]

Jackson, J. M., Rathborne, J. M., Shah, R. Y., et al. 2006, ApJS, 163,145 [1]

Jones, C., \& Dickey, J. M. 2012, ApJ, 753, 62 [1]

Kauffmann, J., Pillai, T., Shetty, R., Myers, P. C., \& Goodman, A. A. 2010, ApJ, 716, 433 [1, 5.3]

Kennicutt, Jr., R. C. 1998, ApJ, 498, 541 [1, 6.1, 6.1, 7.1]

Koda, J., Scoville, N., Hasegawa, T., et al. 2012, ApJ, 761, 41 [4.1]

Koyama, H., \& Inutsuka, S. 2000, ApJ, 532, 980 [7.3]

Krumholz, M. R., \& Burkhart, B. 2016, MNRAS, 458, 1671 [6.3, 7.3]

Krumholz, M. R., Dekel, A., \& McKee, C. F. 2012a, ApJ, 745, 69 [1]

-. 2012b, ApJ, 745, 69 [6.1]

Lada, C., Dickinson, D. F., \& Penfield, H. 1974, ApJ, 189, L35 [1]

Lada, C. J., Forbrich, J., Lombardi, M., \& Alves, J. F. 2012, ApJ, $745,190 \quad[1,6.2]$

Lada, C. J., Lombardi, M., \& Alves, J. F. 2010, ApJ, 724, 687 $[2.3,4.2,6.2,7.1]$

Langer, W. D., Velusamy, T., Pineda, J. L., Willacy, K., \& Goldsmith, P. F. 2014, A\&A, 561, A122 [4.1]

Larson, R. B. 1981, MNRAS, 194, 809 [1, 5.1]

Leroy, A. K., Walter, F., Sandstrom, K., et al. 2013, AJ, 146, 19 $[2.3]$

Lombardi, M., Alves, J., \& Lada, C. J. 2010, A\&A, 519, L7 [1, 5.3] 
Louvet, F., Motte, F., Hennebelle, P., et al. 2014, A\&A, 570, A15 $[1,7.3]$

Martins, F., Schaerer, D., \& Hillier, D. J. 2005, A\&A, 436, 1049 $[4.2]$

Maruta, H., Nakamura, F., Nishi, R., Ikeda, N., \& Kitamura, Y. 2010, ApJ, 714, 680 [2.3, 6]

Mezger, P. G., \& Henderson, A. P. 1967, ApJ, 147, 471 [4.2]

Miura, R. E., Kohno, K., Tosaki, T., et al. 2012, ApJ, 761, 37 [2.3]

-. 2014, ApJ, 788, 167 [2.3, 4.2]

Motte, F., Schilke, P., \& Lis, D. C. 2003, ApJ, 582, 277 [1]

Motte, F., Nguyen-Luong, Q., Schneider, N., et al. 2014, A\&A, 571, A32 [7.3]

Murray, N. 2011, ApJ, 729, 133 [2.3, 4.1, 7.2]

Murray, N., \& Rahman, M. 2010, ApJ, 709, 424 [4.2]

Nguyen, H., Nguyen-Luong, Q., Martin, P. G., et al. 2015, ApJ, $812,7 \quad[3,4.2,7.2]$

Nguyen Luong, Q., Motte, F., Hennemann, M., et al. 2011a, A\&A, 535, A76 [3, 4.1, 7.2]

Nguyen Luong, Q., Motte, F., Schuller, F., et al. 2011b, A\&A, $529, \mathrm{~A} 41+[1,7.2,7.3]$

Nguyen-Luong, Q., Motte, F., Carlhoff, P., et al. 2013, ApJ, 775, 88 [7.3]

Onishi, T., Mizuno, A., Kawamura, A., Tachihara, K., \& Fukui, Y. 2002, ApJ, 575, 950 [2.3, 6]

Portegies Zwart, S. F., McMillan, S. L. W., \& Gieles, M. 2010, ARA\&A, 48, $431 \quad[1,4.1,2]$

Rebolledo, D., Wong, T., Xue, R., et al. 2015, ApJ, 808, 99 [1, 5.1]

Reid, M. J., Menten, K. M., Zheng, X. W., et al. 2009, ApJ, 700, $137[1]$

Reid, M. J., Menten, K. M., Brunthaler, A., et al. 2014, ApJ, 783, 130 [3]

Robitaille, T. P., \& Whitney, B. A. 2010, ApJ, 710, L11 [7.2]

Rodgers, A. W., Campbell, C. T., \& Whiteoak, J. B. 1960, MNRAS, 121, 103 [7.2]

Roman-Duval, J., Jackson, J. M., Heyer, M., Rathborne, J., \& Simon, R. 2010, ApJ, 723, 492 [2.3, 4.1, 5.3]

Rosolowsky, E. 2007, ApJ, 654, 240 [2.3]

Rosolowsky, E. W., Pineda, J. E., Kauffmann, J., \& Goodman, A. A. 2008, ApJ, 679, 1338 [3]

Rygl, K. L. J., Brunthaler, A., Sanna, A., et al. 2012, A\&A, 539, A79 [1]

Sakamoto, K., Wang, J., Wiedner, M. C., et al. 2008, ApJ, 684, $957[7.2]$

Sanna, A., Reid, M. J., Menten, K. M., et al. 2014, ApJ, 781, 108 [1]

Sato, M., Reid, M. J., Brunthaler, A., \& Menten, K. M. 2010, ApJ, 720, 1055 [1]

Schmidt, M. 1959, ApJ, 129, 243 [1]
Schneider, N., Bontemps, S., Simon, R., et al. 2006, A\&A, 458, $855[4.1,7.2]$

Schneider, N., \& Brooks, K. 2004, PASA, 21, 290 [1]

Scoville, N., Sheth, K., Walter, F., et al. 2015, ApJ, 800, 70 [7.2]

Scoville, N. Z. 2013, Evolution of star formation and gas, ed. J. Falcón-Barroso \& J. H. Knapen, 491 [2.3]

Shetty, R., Glover, S. C., Dullemond, C. P., et al. 2011, MNRAS, 415,3253 [4.1]

Shimajiri, Y., Kitamura, Y., Nakamura, F., et al. 2015, ApJS, $217,7 \quad[2.3,6]$

Simon, R., Jackson, J. M., Clemens, D. P., Bania, T. M., \& Heyer, M. H. 2001, ApJ, 551, 747 [4.1]

Soifer, B. T., Neugebauer, G., Helou, G., et al. 1984, ApJ, 283, L1 [7.2]

Stil, J. M., Taylor, A. R., Dickey, J. M., et al. 2006, AJ, 132, 1158 $[2.2]$

Stutzki, J., \& Guesten, R. 1989, NASA STI/Recon Technical Report N, 90 [3]

Tacconi, L. J., Neri, R., Genzel, R., et al. 2013, ApJ, 768, 74 [2.3]

Taylor, A. R., Gibson, S. J., Peracaula, M., et al. 2003, AJ, 125, $3145[2.2]$

Utomo, D., Blitz, L., Davis, T., et al. 2015, ApJ, 803, 16 [1, 5.1]

Vallée, J. P. 2014, ApJS, 215, 1 [2]

Vazquez-Semadeni, E., Passot, T., \& Pouquet, A. 1996, ApJ, 473, 881 [7.3]

Walsh, A. J., Beuther, H., Bihr, S., et al. 2016, MNRAS, 455, 3494 [3]

Wei, L. H., Keto, E., \& Ho, L. C. 2012, ApJ, 750, 136 [2.3]

Whiting, M., \& Humphreys, B. 2012, PASA, 29, 371 [3]

Whitmore, B. C., Brogan, C., Chandar, R., et al. 2014, ApJ, 795, 156 [1]

Williams, J. P., de Geus, E. J., \& Blitz, L. 1994, ApJ, 428, 693 [3]

Willis, S., Guzman, A., Marengo, M., et al. 2015, ApJ, 809, 87 $[1,6.1]$

Wilson, C. D., Rangwala, N., Glenn, J., et al. 2014, ApJ, 789, L36 [7.2]

Wilson, R. W., Jefferts, K. B., \& Penzias, A. A. 1970, ApJ, 161, L43 [1]

Wilson, T. L., Casassus, S., \& Keating, K. M. 2012, ApJ, 744, $161[4.2]$

Wu, J., Evans, II, N. J., Gao, Y., et al. 2005, ApJ, 635, L173 $[6.2,7.1]$

Xu, Y., Moscadelli, L., Reid, M. J., et al. 2011, ApJ, 733, 25 [1]

Zanella, A., Daddi, E., Le Floc'h, E., et al. 2015, Nature, 521, 54 $[2.3,7.2]$

Zhang, B., Reid, M. J., Menten, K. M., et al. 2013, ApJ, 775, 79 [1]

Zhang, B., Zheng, X. W., Reid, M. J., et al. 2009, ApJ, 693, 419 [1]

Zhang, B., Moscadelli, L., Sato, M., et al. 2014, ApJ, 781, 89 [1] 